\title{
Context-dependent modulation of aggressiveness of pediatric tumors by individual oncogenic RAS isoforms
}

\author{
Julia Bauer (iD) ${ }^{1}$, Nicole Cuvelier ${ }^{1}$, Nada Ragab ${ }^{1}$, Katja Simon-Keller ${ }^{2}$, Frauke Nitzki ${ }^{1}$, Natalie Geyer ${ }^{1}$, Dominik S. Botermann (iD ${ }^{1}$, \\ Dominik P. Elmer (iD ${ }^{3}$, Albert Rosenberger (iD) ${ }^{4}$, Thomas A. Rando ${ }^{5}$, Stefano Biressi ${ }^{5,6,7}$, James A. Fagin (iD ${ }^{8}$, Dieter Saur (iD ${ }^{9}$,

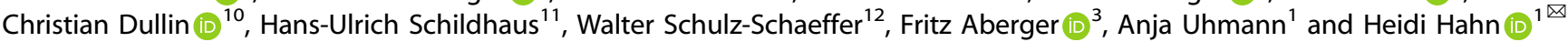

(c) The Author(s) 2021

\begin{abstract}
A prototypic pediatric cancer that frequently shows activation of RAS signaling is embryonal rhabdomyosarcoma (ERMS). ERMS also show aberrant Hedgehog $(\mathrm{HH}) / \mathrm{GLI}$ signaling activity and can be driven by germline mutations in this pathway. We show, that in ERMS cell lines derived from sporadic tumors i.e. from tumors not caused by an inherited genetic variant, $\mathrm{HH} / \mathrm{GLI}$ signaling plays a subordinate role, because oncogenic mutations in HRAS, KRAS, or NRAS (collectively named oncRAS) inhibit the main HH target GLI1 via the MEK/ERK-axis, but simultaneously increase proliferation and tumorigenicity. oncRAS also modulate expression of stem cell markers in an isoform- and context-dependent manner. In Hh-driven murine ERMS that are caused by a Patched mutation, oncHRAS and mainly oncKRAS accelerate tumor development, whereas oncNRAS induces a more differentiated phenotype. These features occur when the oncRAS mutations are induced at the ERMS precursor stage, but not when induced in already established tumors. Moreover, in contrast to what is seen in human cell lines, oncRAS mutations do not alter Hh signaling activity and marginally affect expression of stem cell markers. Together, all three oncRAS mutations seem to be advantageous for ERMS cell lines despite inhibition of $\mathrm{HH}$ signaling and isoform-specific modulation of stem cell markers. In contrast, oncRAS mutations do not inhibit Hhsignaling in Hh-driven ERMS. In this model, oncRAS mutations seem to be advantageous for specific ERMS populations that occur within a specific time window during ERMS development. In addition, this window may be different for individual oncRAS isoforms, at least in the mouse.
\end{abstract}

Oncogene (2021) 40:4955-4966; https://doi.org/10.1038/s41388-021-01904-4

\section{INTRODUCTION}

Rhabdomyosarcoma (RMS) is the most common type of soft tissue sarcoma in children with poor prognosis [1]. The major pediatric form is embryonal RMS (ERMS), which accounts for $\sim 75 \%$ of RMS (reviewed by e.g. [2]). ERMS originate from muscle progenitor or stem cells [3-5] and contain cell populations with tumorpropagating or cancer stem cell (CSC) features (for review see ref. [6]) that may explain their intratumoral heterogeneity $[7,8]$.

ERMS is a prototypic RAS-associated pediatric cancer. Indeed, individual oncogenic RAS (oncRAS) mutations affecting all three RAS genes (HRAS, KRAS, and NRAS) occur in up to $42 \%$ of ERMS [9-11]. The current discussion hinges on whether oncRAS mutations are ERMS drivers or rather modifiers. In favor of the "driver-hypothesis" are studies in zebrafish [12] and genomic analyses including whole genome sequencing analysis [7, 10, 11]. Moreover, patients with Noonan or Costello syndrome, which are caused by activating $K_{-}, N$ - or HRAS germline mutations, respectively, are predisposed to ERMS [13]. On the other hand, microarray-based data showing that a RAS signature exists together only with signatures from other activated signaling pathways [5] and the fact that oncRAS mutations are found in ERMS only in combination with other mutations and do not lead to ERMS when occuring alone in the mouse [14-17] favor the "modifier-hypothesis". Altogether oncRAS mutations seem to play a very important role in ERMS pathogenesis although their exact role still remains to be clarified.

Another pathway that is active in ERMS is Hedgehog $(\mathrm{HH})$ signaling [18-20]. The major players of $\mathrm{HH}$ signaling are the $\mathrm{HH}$ ligands, the $\mathrm{HH}$ receptor Patched1 (PTCH), the PTCH interaction partner Smoothened (SMO) and GLI transcription factors. Aberrant activation of $\mathrm{HH}$ signaling leads to a variety of tumors including ERMS (reviewed by e.g., ref. [21]). Indeed, inherited PTCH/Ptch mutations can result in ERMS formation both in humans and mice $[22,23]$. The most reliable read-out of the pathway's activity is the

\footnotetext{
${ }^{1}$ Department of Human Genetics, University Medical Center Goettingen, Goettingen, Germany. ${ }^{2}$ Institute of Pathology, University Medical Center Mannheim, University of Heidelberg, Mannheim, Germany. ${ }^{3}$ Department of Biosciences, Paris-Lodron University of Salzburg, Cancer Cluster Salzburg, Salzburg, Austria. ${ }^{4}$ Department of Genetic Epidemiology, University Medical Center Goettingen, Goettingen, Germany. ${ }^{5}$ Paul F. Glenn Center for the Biology of Aging and Department of Neurology and Neurological Sciences, Stanford University School of Medicine, Stanford, CA, USA. ${ }^{6}$ Neurology Service, Veterans Affairs Palo Alto Health Care System, Palo Alto, CA, USA. ${ }^{7}$ Department of Cellular, Computational and Integrative Biology (CIBIO) and Dulbecco Telethon Institute, University of Trento, Povo-Trento, Italy. ${ }^{8}$ Memorial Sloan Kettering Cancer Center, New York, NY, USA. ${ }^{9}$ Klinik und Poliklinik für Innere Medizin II, Klinikum rechts der Isar der TUM, Muenchen, Germany. ${ }^{10}$ Institute for Diagnostic and Interventional Radiology, University

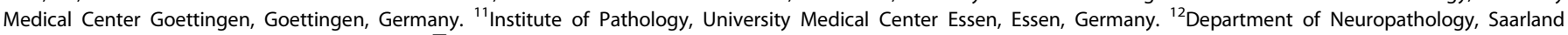
University Medical Center, Homburg, Germany. ${ }^{凶}$ email: hhahn@gwdg.de
}

Received: 29 September 2020 Revised: 25 May 2021 Accepted: 9 June 2021

Published online: 25 June 2021 
4956

transcriptional level of GLI1 (reviewed by e.g., refs. [24, 25]. We and others found that the expression of GLI1 and other $\mathrm{HH}$ targets is higher in ERMS compared to alveolar RMS (ARMS) [18, 19]. However, sporadic ERMS lack canonical HH signaling activity via the $\mathrm{HH} / \mathrm{PTCH} / \mathrm{SMO} / \mathrm{GLI}$ axis $[26,27]$ and GLI activity is apparently regulated in a non-canonical manner, which summarizes the regulation of GLI transcription factors by interaction with other signaling pathways including the RAS pathway (reviewed by e.g., refs. $[24,28]$.

Here we compared the influence of oncH-, oncK-, and oncNRAS mutations on ERMS growth in different experimental settings using human ERMS cell lines derived from sporadic ERMS and the $\mathrm{Ptch}^{+/-}$mouse model that develops ERMS-like tumors due to inherited Ptch mutations [23]. This allowed us to investigate the impact of oncRAS mutations on early and late ERMS stages and also on canonical or non-canonical HH/GLI-signaling in ERMS.

\section{RESULTS \\ OncRAS mutations can inhibit GLI1/GLI1 expression via the MEK/ERK axis in human ERMS cell lines}

In order to investigate the impact of oncRAS mutations on noncanoncial $\mathrm{HH} / \mathrm{GLI}$ signaling activity in established human sporadic ERMS, the RAS wildtype ERMS cell lines RUCH-2 and TE617.T were stably transduced with pMSCVpuro-HRAS ${ }^{G 12 V}$, pMSCVpuro-KRAS ${ }^{G 12 V}$, pMSCVpuro-NRAS ${ }^{G 12 V}$, or the pMSCVpuro empty vector (HRAS, KRAS, NRAS, or pMSCV, respectively). DNA integration and CDNA expression was demonstrated by PCR and RAS protein expression (Fig. $1 \mathrm{~A}, \mathrm{~B} ; \mathrm{KRAS}^{\mathrm{G} 12 \mathrm{~V}}$ is $\mathrm{HA}$-tagged and is larger than the endogenous KRAS protein). Unfortunately, HRAS and NRAS were expressed in the same TE617.T cell clone, probably due to inadvertent transduction with both vectors (Fig. 1B). Elevated RAS activity was verified by RAS activation assay (Fig. 1A, B).

Our results show that oncRAS mutations can negatively regulate GLI1 mRNA expression in RUCH-2 and TE617.T (Fig. 1C, D; results for TE617.T H-/NRAS are not significant). To investigate whether the two main RAS-downstream pathways are involved in suppression of GLI1, the cells were incubated with the AKT/ mTOR inhibitor PI-103, the MEK inhibitor U0126 or the ERK inhibitor SCH772984 (see Fig. S1A-C for inhibitor functionality). Whereas PI-103 downregulated GLI1 in both control and oncRAStransduced RUCH-2 cells (Fig. 1E;), incubation of RUCH-2 oncRAS cells with U0126 or SCH772984 restored GLI1 expression to basal levels of control cells (Fig. 1E). This was also seen in RD cells (Fig. S1B) that harbor a NRAS ${ }^{\mathrm{Q} 61 \mathrm{H}}$ mutation [29]. In TE617.T cells, PI-103 had no effect and MEK and/or ERK inhibit GLI1 expression independently of oncRAS, because U0126 and SCH772984 elevated GLI1 also in control cells (Fig. 1F). This might be due to mutations in MAP3K14/NIK or MAP3K1/MEKK1 of TE617.T cells, which result in an alternative splice variant or a threonine deletion at position 949, respectively (The Cancer Cell Line Encyclopedia [30]). The function of these changes is unknown. However, because these proteins can activate ERK [31, 32], these changes may have influenced the activity of ERK. Indeed, SCH772984-mediated pERK suppression was weaker compared to RUCH-2 cells (Fig. S1C).

To confirm that ERK is involved in GLI1 suppression, ERK1 and/or ERK2 expression was decreased in RUCH-2 KRAS cells by transient siRNA transfection. Indeed, even a partial ERK1 and/or ERK2 knockdown restores GLI1 expression to basal levels of pMSCV control cells (Fig. 2A; not completely restored by ERK2 knockdown), indicating that ERK suppresses GLI1. The data also show that the ERK knockdown does not influence PI-103-mediated downregulation of GLI1 (Fig. 2A). This implicates that the AKT axis rather activates GLI1 expression, at least in RUCH-2 KRAS cells. However, this situation is certainly much more complex, because in dependency of the cell line (i) PI-103-mediated GLI1 suppression is associated with ERK phosphorylation (Fig. 2A; Fig. S1A-C), (ii) ERK1 and ERK2 can influence phosphorylation of each other (Fig. 2A), and (iii) U0126 and SCH772984 can impact AKT phosphorylation (Fig. S1A-C).

We also analyzed whether oncRAS alters the intracellular distribution of GLI1. However, oncRAS downregulated GLI1 protein regardless of the cellular compartment and SCH772984 upregulated GLI1 protein back to normal levels (Fig. 2B).

Together, oncRAS can suppress GL/1/GLI1 expression and thus $\mathrm{HH}$ signaling in ERMS, which can involve the MEK/ERK axis.

\section{Despite attenuation of $\mathrm{HH}$ signaling activity, oncRAS mutations can increase proliferation and tumorigenicity of human ERMS cell lines}

Next, we investigated the impact of oncRAS mutations on ERMS growth. BrdU incorporation in a timeframe of $72 \mathrm{~h}$ revealed a significant increase in proliferation of oncHRAS- and oncKRAS-, but not of oncNRAS-expressing RUCH-2 cells (Fig. 3A, left panel). Cell viability was not affected (Fig. 3A right panel). In TE617.T cells, proliferation rate and cell viability were significantly increased upon oncKRAS or oncH-/NRAS expression as measured in a timeframe of $24 \mathrm{~h}$ by BrdU incorporation and WST-1 assays, respectively (Fig. 3B). Cellular appearance was never affected (Fig. S1D). To evaluate the in vivo growth behavior, control and oncRAS cell lines were transplanted into nude mice. Indeed, all oncRAS cell lines including oncNRAS-expressing RUCH-2 cells showed a significantly accelerated growth and end point weight (Fig. 3C, D). In general, GLI1 expression remained downregulated compared to the corresponding control xenotransplants. GLI1downregulation was significant for oncKRAS-expressing RUCH-2 and oncH-/NRAS expressing TE617.T xenotransplants (Fig. 3C, D). Although the exact roles of oncH- and oncNRAS in TE617.T remains to be determined, the data demonstrate that oncRAS can accelerate proliferation and tumorigenicity of ERMS cell lines despite downregulation of $\mathrm{HH}$ signaling.

\section{OncRAS mutations can alter the expression of stem cell markers of human ERMS cell lines in an isoform- and context- dependent manner}

Because oncRAS can change the expression of cancer stem cell (CSC) markers [33], oncRAS-expressing RUCH-2 were analyzed in a pilot experiment for the activity of aldehyde dehydrogenase (ALDH) that is associated with self-renewal and tumor formation capacity in RD cells [34]. Indeed, as revealed by Aldeflour assay, oncRAS-expressing RUCH-2 cells showed a slight, but not significant increase in ALDH ${ }^{\text {high }}$ cells compared to control cells (Fig. 4A). This was also seen on protein level (Fig. 4B). Next, we analyzed 84 CSC-associated genes of a commercially available RT-PCR array. Using arbitrary fold change cut-offs of $>2$ and $<2$, all oncRAS RUCH-2 cells showed upregulated CD34, CXCL8, ITGA6, LIN28B, MYC, TGFBR1, and WWC1, and downregulated $A L C A M, B M P 7$, and DLL1 expression (Fig. 4C). All other genes did not meet the cut-off criteria or were differentially regulated by individual oncRAS isoforms like SOX2, which was upregulated by oncKRAS and oncHRAS, but not by oncNRAS (Fig. 4C). To investigate whether this expression pattern was retained after transplantation, MYC or SOX2 expression of oncKRAS and oncNRAS RUCH-2 xenotransplants was examined by qRT-PCR or immunhistological stainings, respectively. These approaches showed that MYC expression was no longer significantly elevated after transplantation (Fig. 4D) and that oncNRAS xenotransplants started to expressed SOX2 (Fig. 4E). The latter observation could explain why oncNRAS expressing RUCH-2 cells robustly grew in vivo (Fig. $3 \mathrm{C}$ ), but hardly grew in vitro (Fig. 3A, right panel). Together, although the data are very preliminary, the experiments implicate that oncRAS modulate expression of CSC-associated genes in an isoform- and contextdependent manner. 
A

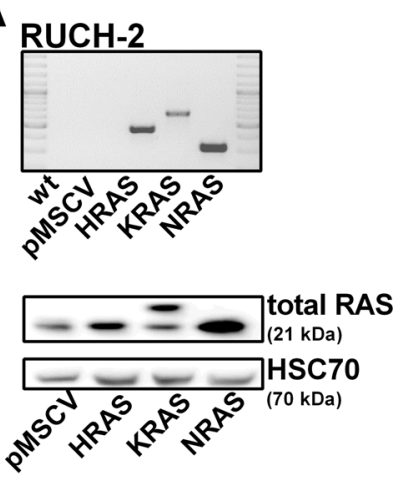

B
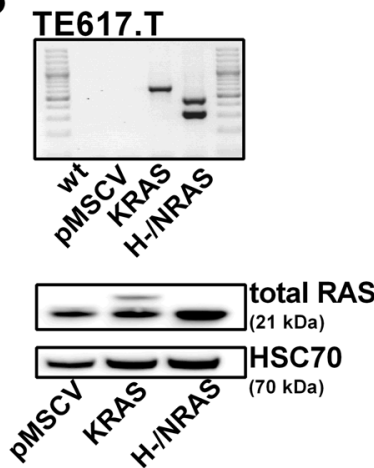

C
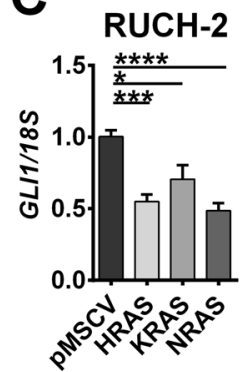

D

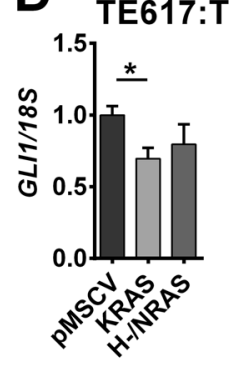

E
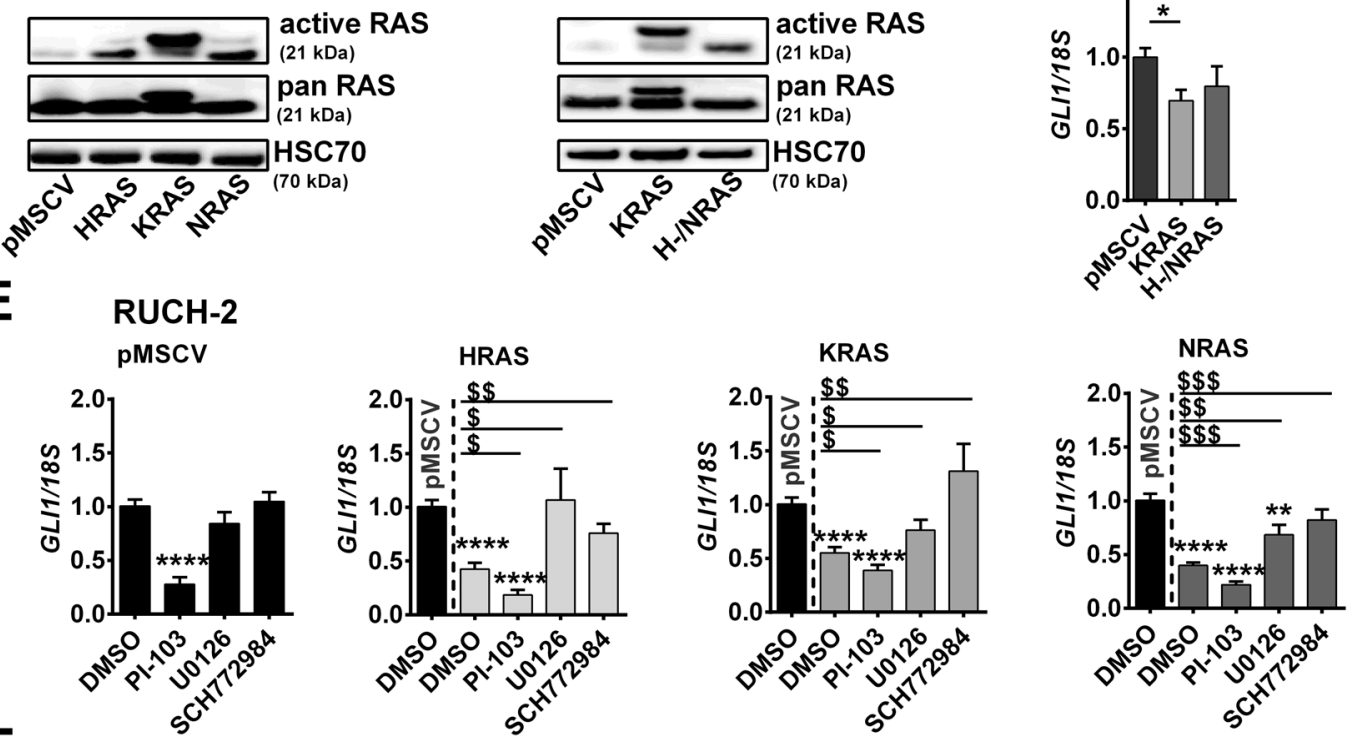

$\mathbf{F}$
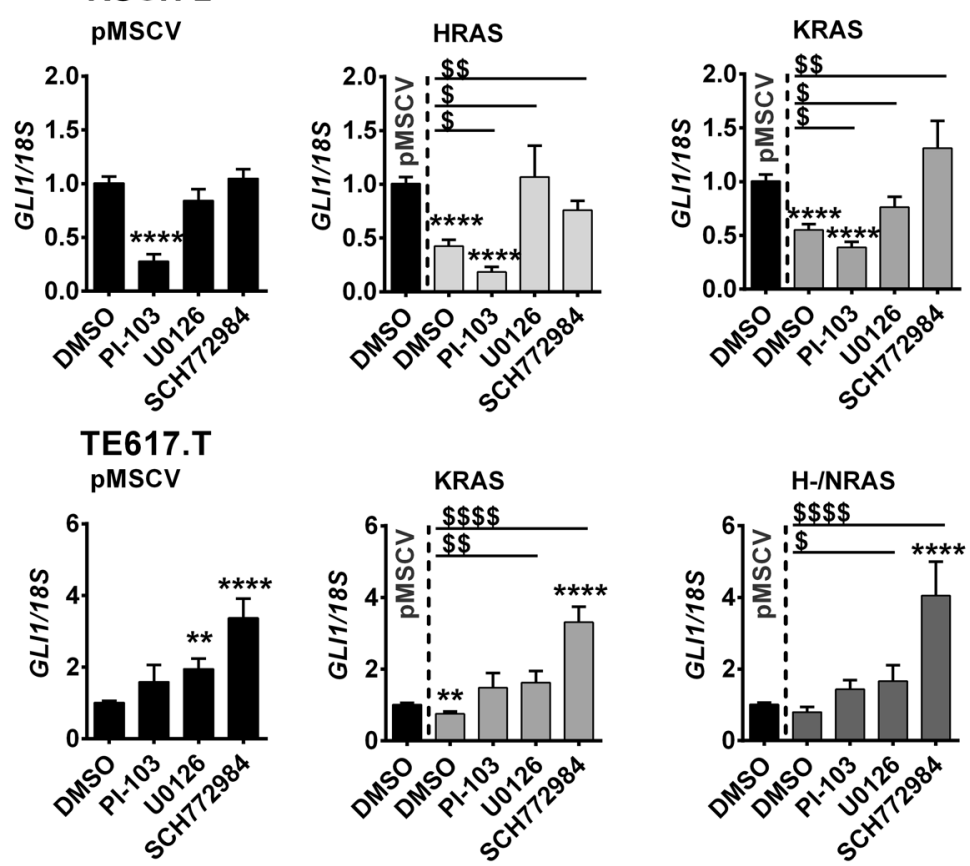

Fig. 1 Impact of oncRAS mutations on GLI1 expression in RUCH-2 and TE617.T ERMS cells. A, B Expression of HRAS, KRAS, NRAS, or PMSCV was confirmed by RT-PCR on cDNA level (top), western blot analyses for RAS protein (middle) and RAS activity by RAS-GTP pulldown assay $(n=2$, bottom) in (A) RUCH-2 and (B) TE617.T cell lines. HSC70 was used as loading control. C, D GLI1 qRT-PCR analyses of (C) HRAS-, KRAS-, NRAS-expressing RUCH-2 and (D) KRAS- or H-/NRAS-expressing TE617.T cells compared to respective pMSCV control cells. E, F GLI1 qRT-PCR analyses of (E) HRAS-, KRAS- or NRAS-expressing RUCH-2 and F KRAS- or H-/NRAS-expressing TE617.T cells treated with $3 \mu M$ PI-103-, $10 \mu M$ U0126- or $0.5 \mu \mathrm{M}$ SCH772984 compared to pMSCV cells. DMSO-treated $(1 \mu \mathrm{l} / \mathrm{ml})$ cells served as controls. Data are shown as fold induction over the expression level of solvent-treated pMSCV control cells, which was set to 1 . Bars show mean + SEM. ${ }^{*}$ or ${ }^{\$}$ : significant compared to solventtreated pMSCV control or solvent-treated oncRAS cell line tested by Mann-Whitney test. ${ }^{* / \$} p<0.05,{ }^{*} / \$ \$ p<0.01,{ }^{* * * / \$ \$ \$} p<0.001,{ }^{* * * / \$ \$ \$ \$}$ $p<0.0001$.

Without affecting Hh signaling activity, oncHRAS or oncKRAS induction at the ERMS precursor stage in $\mathrm{Ptch}^{+/-}$mice accelerates tumor growth, whereas oncNRAS results in a more differentiated tumor phenotype

To test the influence of oncRAS mutations on growth and $\mathrm{HH}$ signaling activity in Hh-driven ERMS, oncRAS mutations were induced in ERMS of $\mathrm{PtCh}^{+/-}$mice, which are wildtype for Hras, Kras, and Nras (oncRas mutations were excluded by sequencing).
This model also allowed us to study the impact of oncRAS mutations on early and late ERMS stages.

Because ERMS of Ptch ${ }^{+/-}$mice highly express Myf5 [35, 36], the Myf5 ${ }^{\text {CreER }}$ Cre-driver [37] was used to conditionally activate oncRAS mutations in the tumors. The Myf5 ${ }^{\text {CreER }}$ Cre-driver's activity in ERMS and at the 3 Ras loci was confirmed by lineage tracing using Ptch $^{+/-}{ }^{2} 26 R^{+/-}$Myf5 ${ }^{\text {CreER/+ }}$ mice (Fig. S2A) and by specific recombination assays (Fig. S2B; ERMS of Ptch ${ }^{+-}$oncRas $^{f /+}{ }^{-1}$ Myf5 $^{\text {CreER/wt }}$ mice 
A

RUCH-2

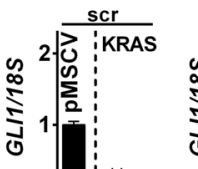

01
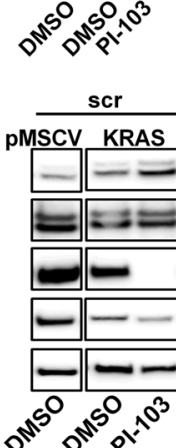
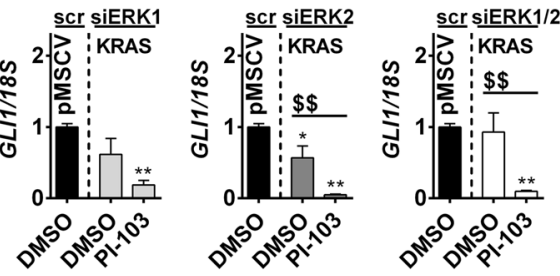

scr siERK1 scr siERK2

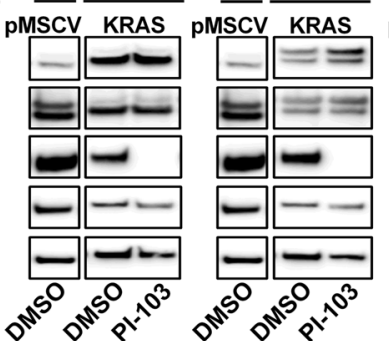

scr siERK1/2 PMSCV KRAS

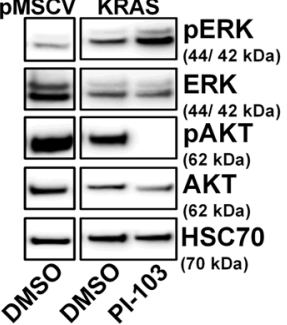

B RUCH-2
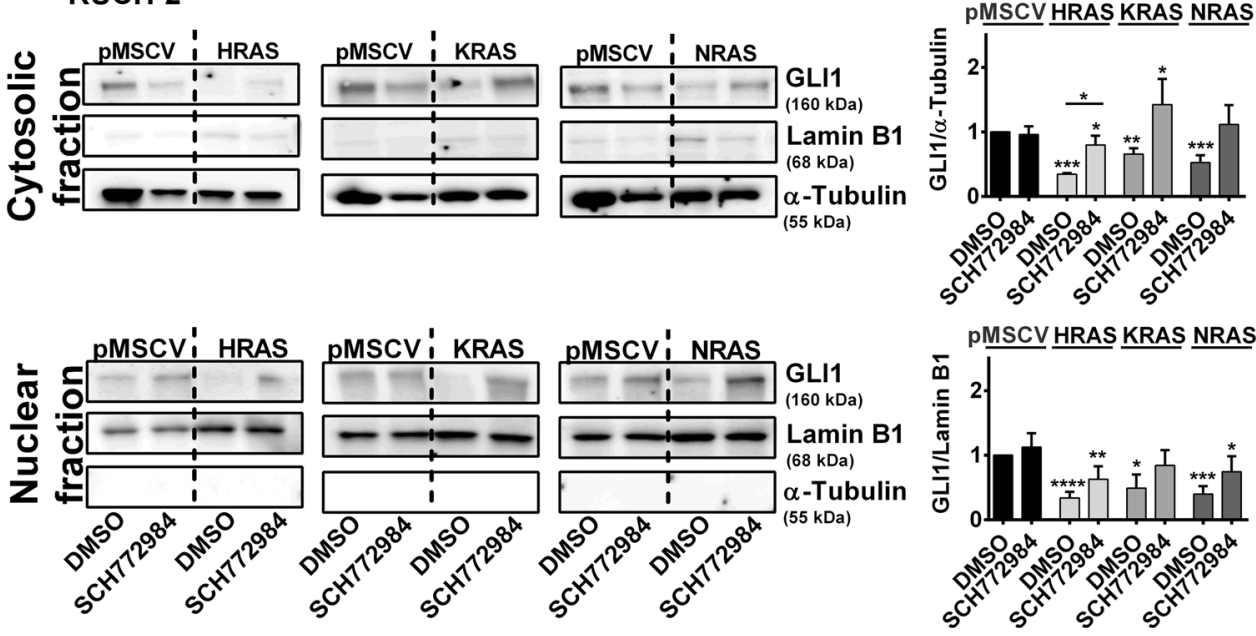

Fig. 2 Impact of ERK on GLI1 expression in RUCH-2 cells. A GLI1 expression (top) and representative pERK/ERK and pAKT/AKT western blot analyses (bottom) $(n=2)$ of KRAS-expressing RUCH-2 cells after siRNA (100 nM each)-mediated ERK1 and/or ERK2 knockdown with and without Pl-103 treatment $(3 \mu \mathrm{M})$ compared to scramble (scr) siRNA transfected KRAS-expressing RUCH-2 and pMSCV control cells. HSC70 served as loading control. B Representative western blot analyses $(n=5)$ (left) and respective densitometric analyses (right) of GLI1 expression in cytosolic and nuclear fractions of HRAS-, KRAS- and NRAS-expressing RUCH-2 cells with or without SCH772984 treatment in comparison to solvent-treated pMSCV control. Lamin B1 or $\alpha$-Tubulin served as loading controls for nuclear or cytosolic fractions, respectively. Bars: mean + SEM. * or $\$$ : significant compared to solvent-treated pMSCV control or solvent-treated oncRAS cell line tested by Mann-Whitney

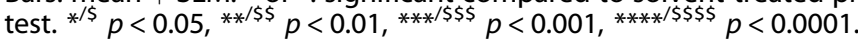

showing spontaneous recombination were excluded from the analyses), respectively. Additionally, tamoxifen-mediated effects on ERMS growth were excluded (Fig. S3A).

Because germline mutations of oncHRAS and oncKRAS or oncNRAS do not result in ERMS or are lethal, respectively [14-17] and because ERMS in $\mathrm{PtCh}^{+/-}$mice are initiated before birth and become palpable at the earliest around 8 weeks of age [36], oncRAS mutations were induced in 4 weeks old $\mathrm{Ptch}^{+/-}$oncRas $^{\mathrm{fl} /+} \mathrm{Myf5}{ }^{\mathrm{CreER} / \mathrm{wt}}$ mice. This allowed for analysis of oncRAS-associated effects on already initiated ERMS precursor lesions.

ERMS development was monitored by palpation for 200 days and sacrificed mice were examined for non-palpable tumors. Compared to the controls, ERMS incidence (palpable and nonpalpable ERMS) was significantly higher in tamoxifen-treated $\mathrm{PtCh}^{+/-} \mathrm{HRas}^{\mathrm{fl} /+} \mathrm{Myf5}^{\mathrm{CreER} / \mathrm{wt}}$ and PtCh ${ }^{+/-} \mathrm{KRas}^{\mathrm{fl} /+} \mathrm{Myf5}^{\mathrm{CreER} / \mathrm{wt}}$ mice (Fig. 5A, B, Table 1). Although both oncHRAS and oncKRAS enforced tumor proliferation (Fig. 5A, B, right panels), only oncKRAS significantly decreased median overall or ERMS-free survival (only palpable ERMS; Fig. 5B, Table 1) and increased tumor multiplicity (mice with $\geq 2$ ERMS; Table 1). In contrast, oncNRAS did not influence any of these parameters (Fig. 5C, Table 1).

Expression of the Hh downstream targets Gli1, Gli2, Ptch1, and Hhip (Fig. S3B-D) and of MyoD and MyHC (Fig. 5D-F) was not changed. However, in contrast to oncHRAS (Fig. 5D) or oncKRAS (Fig. 5E), oncNRAS significantly increased the expression of the early and late differentiation markers Myogenin and Tropomyosin 3 , respectively (Fig. $5 \mathrm{~F}$; see Fig. S3E-G for immunehistochemical analyses of Tropomyosin 3 and $\mathrm{MyHC}$ ). This went along with the appearance of multinucleated cells (Fig. 5G, right panel), which were rarely observed in the other cohorts (Fig. 5G, middle and left panels). 
A

RUCH-2

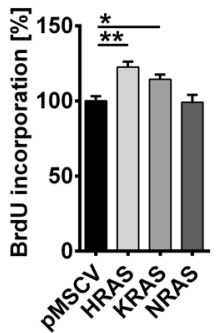

C

RUCH-2

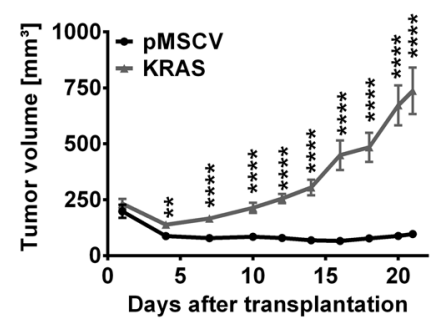

D

TE617.T

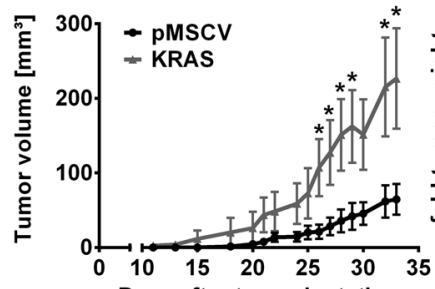

Days after transplantation
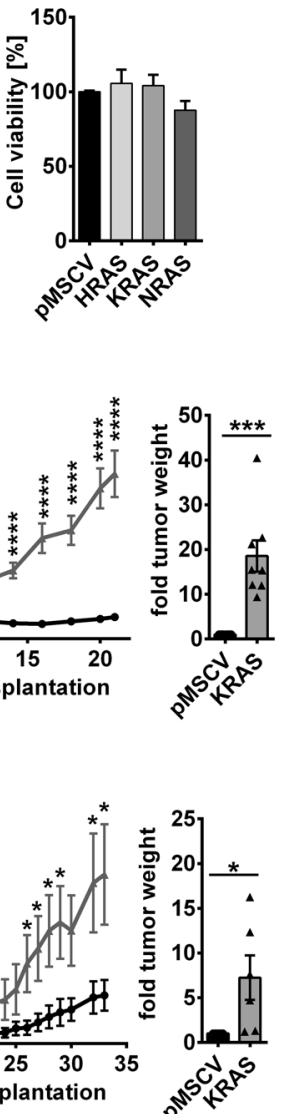

B

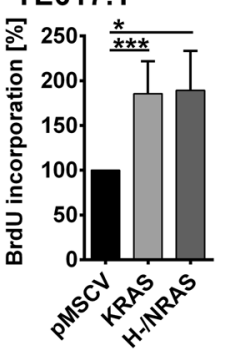

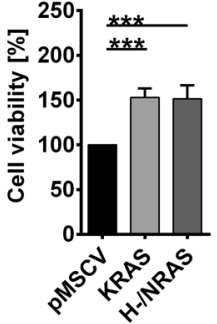

\section{RUCH-2}
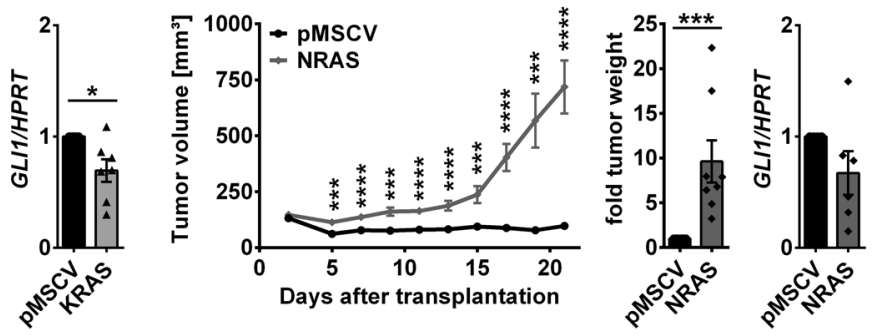

TE617.T

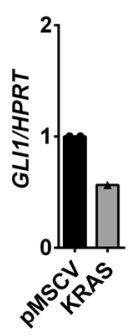

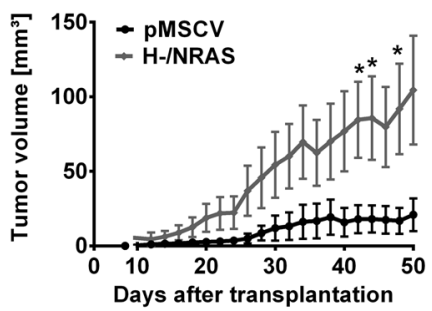
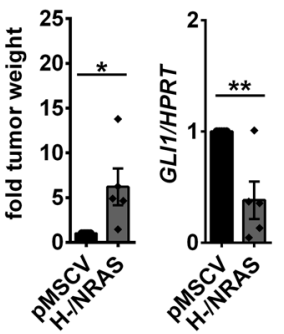

Fig. 3 Impact of oncRAS on growth of RUCH-2 and TE617.T cells and on GLI1 expression after xenotransplantation. A, B BrdU-incorporation (left) and WST-1 cell viability (right) assays of A RUCH-2 $(n=3)$ and B TE617.T $(n=7)$ cells stably expressing HRAS, KRAS, NRAS or H-/NRAS. C, D Mean tumor volume ( \pm SEM), -fold tumor weight and -fold GLI1 expression of C RUCH-2 and D TE617.T xenotransplants expressing KRAS ( $n=8$ mice), NRAS ( $n=8$ mice) or H-/NRAS ( $n=6$ mice) compared to respective pMSCV control tumors of the same mice (controls were all set to 1 for -fold tumor weight and -fold GLI1 expression). Bars: mean + SEM. *Significant by multiple unpaired t-test (tumor growth curve) or Mann-Whitney test (BrdU and WST assay, tumor weight, GLI1 expression) in comparison to pMSCV controls. ${ }^{*} p<0.05,{ }^{* *} p<0.01,{ }^{* * *} p<0.001$, $* * * * 0.0001$.

These data indicate that ERMS precursor lesions of $\mathrm{Ptch}^{+/-}$mice react differently to the induction of oncRAS isoforms. Thus, oncHRAS and oncKRAS mutations reinforce development of fullblown tumors, with oncKRAS being more aggressive. In contrast, oncNRAS apparently induces a more differentiated phenotype.

\section{OncRAS mutations do not alter growth of already established} ERMS of Ptch $^{+/-}$mice

Next, oncRAS mutations were induced in mice with palpable tumors $(\sim 0.5 \mathrm{~cm}$ diameter). As measured by $\mu \mathrm{CT}$ the sizes of all ERMS of the Ptch ${ }^{+/}$oncRas $^{f /+}$ Myf5 $5^{\text {CreER/wt }}$ cohorts were almost identical at onset of the study and after 7 weeks all tumors had grown (Fig. 6A-C, left panels). However, despite efficient Cremediated recombination (Fig. S2B) and enhanced intratumoral RAS activity (Fig. S4A), none of the oncRAS isoforms influenced ERMS growth (Fig. 6A-C; left panels). This was confirmed by the relative increase of individual tumor sizes and by Ki67 expression (Fig. $6 \mathrm{~A}-\mathrm{C}$, middle and right panels). Moreover, the mutations had no impact on the expression of $\mathrm{Hh}$ and differentiation markers (Fig. S4B-D). Tamoxifen-mediated effects on tumor growth (Fig. S5 upper panel), expression of Gli1 and on myogenic differentiation markers (Fig. S5 lower panel) were also excluded.

These results suggest that none of the three oncRAS isoforms influences growth, proliferation or molecular characteristics of fullblown ERMS of Ptch $^{+/-}$mice.
OncRAS mutations do not affect expression of selected stem cell markers in ERMS of Ptch $^{+/-}$mice

To test if oncRAS also regulates the expression of CSC genes in ERMS of Ptch $^{+/-}$mice, the intratumoral protein level of ALDH1A1 was analyzed. ALDH1A1 protein expression was heterogeneous and did neither correlate with oncRAS induction the precursor stage, (Fig. 7A) nor with induction at full-blown tumor stage (Fig. 7B). Equally, the CSC markers Cd34, Itga6, Myc, and Tgfbr1 that were upregulated by oncRAS in human ERMS cell lines, showed no significant differences compared to the controls (Fig. 7C, oncRASexpressing ERMS derived from precursor lesions; Fig. 7D, ERMS that had received the oncRAS mutation at the full-blown stage; please note that Lin28b, CxCl8, and WwC1 were not detected in ERMS or skeletal muscle). Thus, in contrast to human ERMS cell lines, oncRAS mutations rather do not influence the expression of stem cell markers in ERMS of Ptch ${ }^{+/-}$mice, at least not in the bulk of the tumors.

\section{DISCUSSION}

Our data show that oncRAS mutations are advantageous for specific ERMS precursor lesions (murine ERMS model) and ERMS cell lines (human ERMS model) and alter the expression of CSC markers in a context- and isoform-dependent manner. In addition, oncRAS can decrease GLI1/GLI1 expression in cell lines derived 
A

RUCH-2

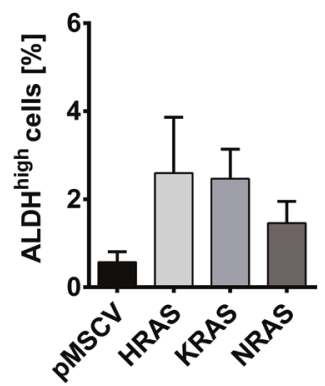

B

RUCH-2

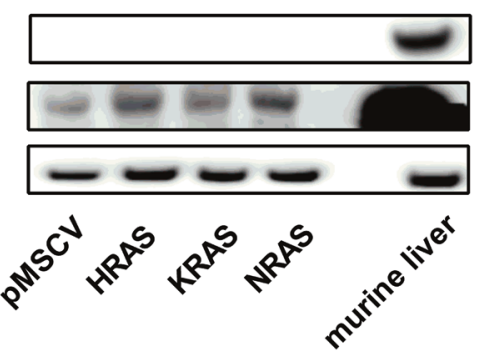

ALDH1A1

(56 kDa, short exp.) ALDH1A1

(56 kDa, long exp.)

HSC7O

(70 kDa)

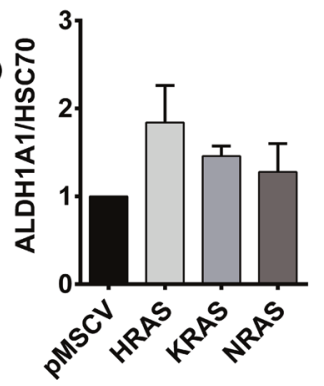

C

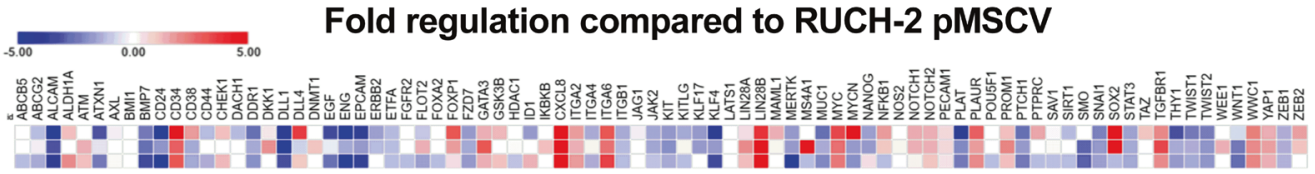

RUCH-2 OncRAS:

$>2$-fold increase: CD34, CXCL8, ITGA6, LIN28B, MYC, TGFBR1, WWC1

$>2$-fold decrease: ALCAM, BMP7, DLL1

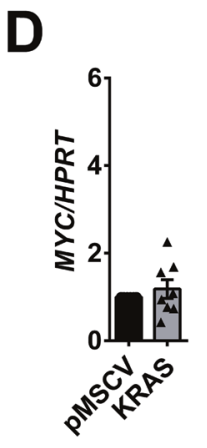

E

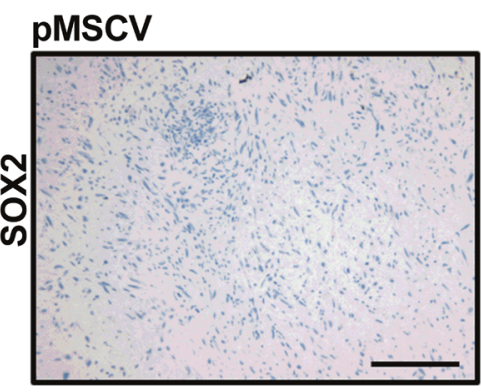

KRAS

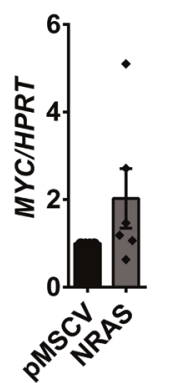

\section{pMSCV}

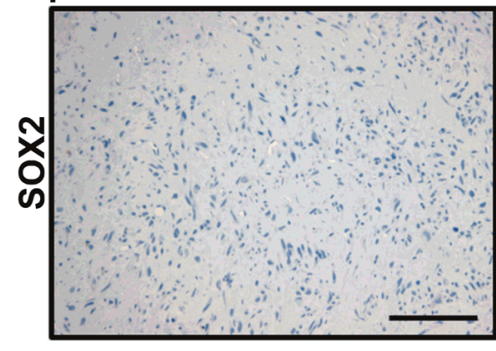

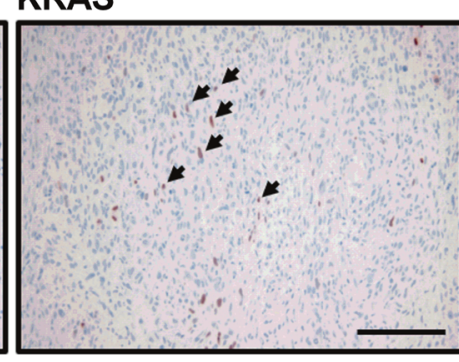

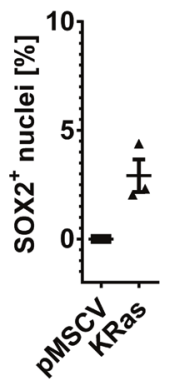

NRAS
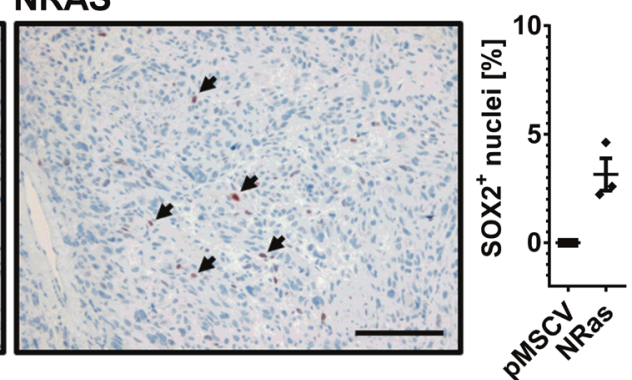

Fig. 4 Impact of oncRAS on expression of stem cell markers in RUCH-2 cells. A Percentage of Aldefluor ${ }^{\text {high }}$ (ALDH $\left.{ }^{\text {high }}\right)$ subpopulations of RUCH-2 cells stably expressing HRAS, KRAS, NRAS or pMSCV $(n=3)$ measured by flow cytometry. B Representative western blot $(n=2)$ (left) and corresponding densitometric analyses (right) of ALDH1A1 expression in HRAS-, KRAS-, and NRAS-expressing RUCH-2 cells in comparison to RUCH-2 pMSCV control cells. Protein lysate of murine liver served as positive control. C Mean fold regulation of 84 cancer stem cellassociated genes in HRAS-, KRAS-, and NRAS-expressing RUCH-2 cells compared to RUCH-2 pMSCV cells $(n=2)$. D MYC qRT-PCR analyses and E anti-SOX 2 antibody stainings and percentage of SOX2 ${ }^{+}$nuclei of KRAS and NRAS-expressing RUCH- 2 xenotransplants $(n=8$ or $n=3$ for $M Y C$ qRT-PCR or SOX2 stainings, respectively) compared to respective pMSCV control tumors of the same mice (set to 1 for MYC qRT-PCR). Bars in A, D: mean + SEM. Scale bars: $100 \mu \mathrm{m}$. Arrows: SOX2 ${ }^{+}$nuclei.

from sporadic ERMS. Because concomitantly cellular proliferation was increased, the data suggest that $\mathrm{HH}$ signaling is not the main driver of growth of sporadic ERMS, although ERMS cell lines are sensitive to the GLI1/2 inhibitor GANT61 [38]. However, oncRAS might override the need for other growth stimuli such as GLI1, because it is a very potent proliferative stimulus. This also has been shown in medulloblastoma, in which oncHRAS circumvents
$\mathrm{HH}$ pathway dependency, drives tumor growth, and enhances metastatic behavior [39].

In ERMS cell lines, the MEK-ERK axis of oncRAS is of great importance for inhibition of GLI1/GLI1 expression. This is similar to a report showing that the MEK-ERK arm is required for an oncKRAS-mediated block of GLI1 expression in fibroblasts and pancreatic carcinoma cell lines [40]. Interestingly, this block needs 
A Ptch $^{+/-H R a s}{ }^{f /+} M y f 55^{C r e E R / w t}$
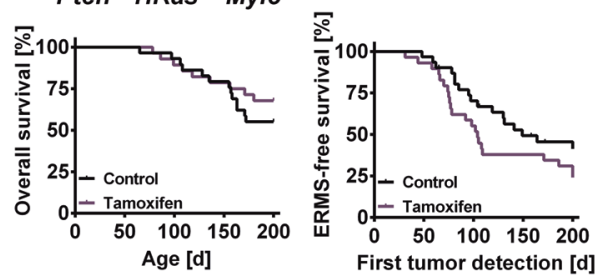

B Ptch $^{+/-}$KRas $^{f / /} M y f 5^{C r e E R / w t}$
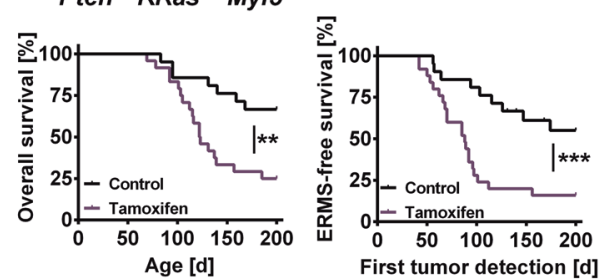

\section{Ptch $^{+/-}$Ras $^{f l /+}$ Myf5 ${ }^{\mathrm{CreER} / w t}$}
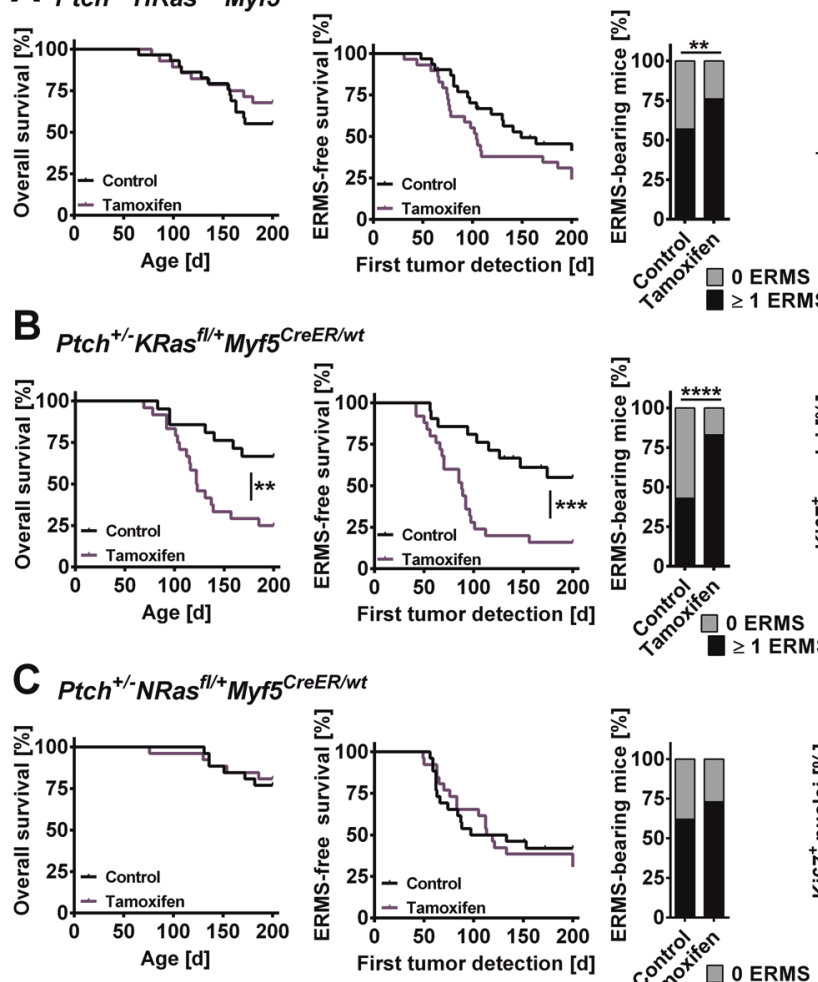
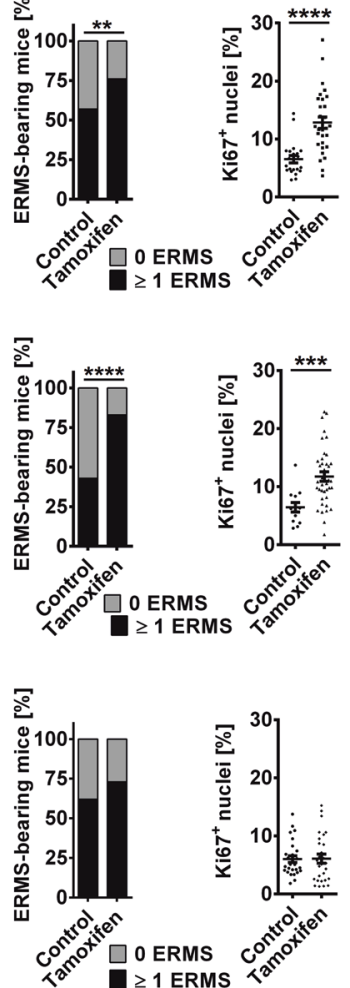

D
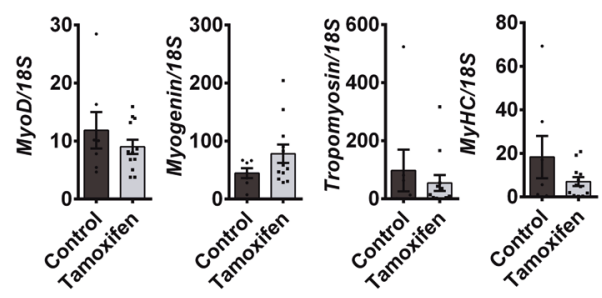

E
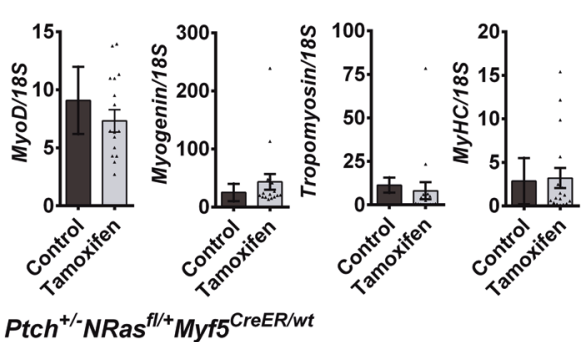

F $\mathrm{Ptch}^{+/-} \mathrm{NRas}^{\mathrm{fl} /+} \mathrm{Myf5}^{\mathrm{CreER} / w t}$
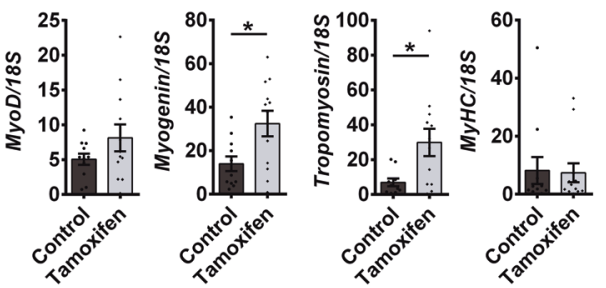

\section{G}
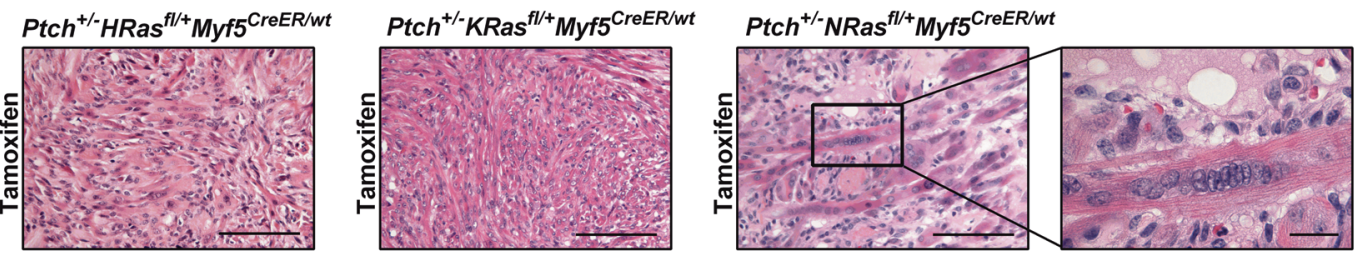

Fig. 5 Impact of oncRAS mutations on progression and differentiation of ERMS precursors in Ptch ${ }^{+/-}$mice. A-C ERMS development in

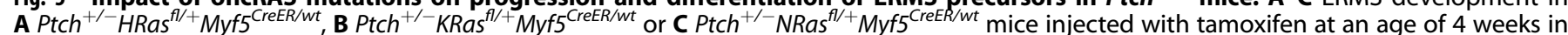
comparison to the control. Numbers of animals and tumors included in the experiments are given in Table 1. From left to right: overall survival, ERMS-free survival (only palpable ERMS), total ERMS incidence (palpable and non-palpable ERMS) and percentage of Ki67 ${ }^{+}$nuclei in ERMS tissue sections. Ki67 staining was done on 10-22 mice of each cohort. Statistical evaluation was done by Log-rank (Mantel-Cox) testing for Kaplan-Meyer curves and by Chi-square testing for tumor incidence. Dots represent the mean percentage of Ki67 $7^{+}$nuclei in individual tumors. D-F qRT-PCR analyses of MyoD, Myogenin, Tropomyosin 3 and Myosin heavy chain (MyHC) in ERMS shown as fold expression of the same gene in normal muscle of the same mouse, which was set to 1. G Representative H\&E stainings of ERMS. Close up: multinucleated cells. Scale bars: $100 \mu \mathrm{m}$ or $20 \mu \mathrm{m}$ (close up). For all experiments untreated mice served as controls. Bars: mean \pm SEM; dots: individual tumors. ${ }^{*} p<$ $0.05,{ }^{* *} p<0.01,{ }^{* * *} p<0.001,{ }^{* * * *} p<0.0001$ compared to control Ptch ${ }^{+/-}$oncRas ${ }^{f / /+}$ Myf5 ${ }^{\text {CreER/wt }}$ mice from the respective cohort and tested by non-parametric $t$-tests (Mann-Whitney).

DYRK1B. Because DYRK1B (i) is important for rhabdomyosarcoma growth [41], (ii) can block $\mathrm{HH}$ signaling [40], and (iii) is a novel ERK2 substrate [42], it is possible that ERK represses the $\mathrm{HH}$ pathway via DYRK1B. However, this is pure speculation and remains to be analyzed in the future.

In contrast, Gli1 expression was not suppressed by oncRAS mutations in the Ptch ${ }^{+/-}$model, which to some extent supports the importance of the Hh pathway for ERMS.

In the Ptch ${ }^{+/}$ERMS model and similar to ERMS cell lines, oncHRAS and oncKRAS enforced tumor proliferation when induced at the ERMS precursor stage. However, oncKRAS was more aggressive and additionally decreased ERMS-free survival. Together with the fact that induction of oncNRAS at the early tumor stage did not alter ERMS growth behavior, our results show that the three oncRAS isoforms can have different functions in ERMS pathogenesis.

When induced at the precursor stage in $\mathrm{PtCh}^{+/-}$mice, oncNRAS did not influence ERMS growth and induced differentiation and myogenin expression. This is surprising, because in human ERMS
oncNRAS mutations are rather associated with an aggressive phenotype and are the most frequent oncRAS mutations [10,11]. In addition, the endogenous NRAS ${ }^{\mathrm{Q} 61 \mathrm{H}}$ mutation in human RD cells inhibits myogenic differentiation by repression of myogenin [43]. This discrepancy may reflect species-specific differences in tumor pathobiology. It is also possible that the Nras ${ }^{G 12 D}$ mutation is not functional in $\mathrm{Ptch}^{+/-}$mice. However, this assumption is unlikely because oncNRAS-associated murine ERMS show elevated RAS activity (see Fig. S4A) and because the Nras ${ }^{G 12 D}$ allele induces malignancies in other models (e.g., see ref. [44]). Our data rather argue for the conclusion that oncRAS-associated processes differ from each other in dependency on their occurrence during tumor development. This hypothesis is supported by the fact that none of the oncRAS mutations influenced tumor growth when induced at the advanced tumor stage in the Ptch $^{+/-}$model. Therefore, it is possible that induction of the oncNRAS mutation at a different stage (e.g., at an earlier time point), when the prospective tumor cells are molecularly different and permissive 


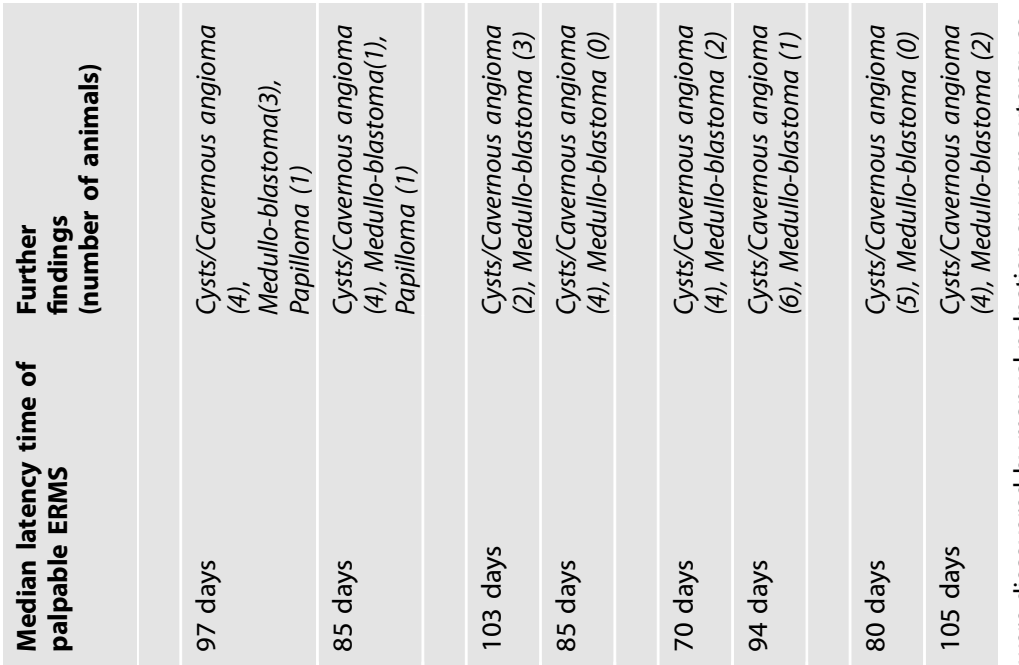

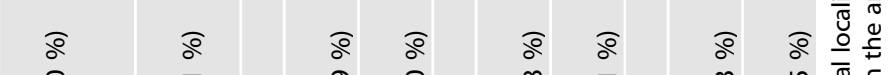

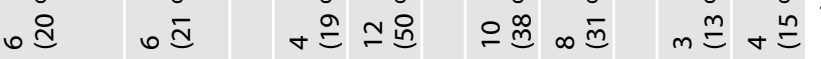

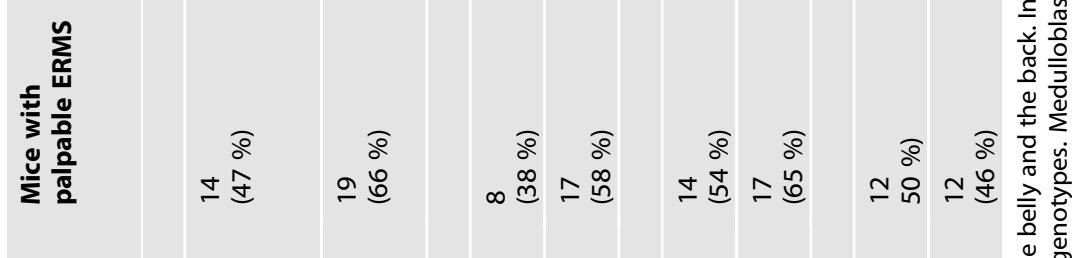

$\sum_{\text {色 }}^{n}$

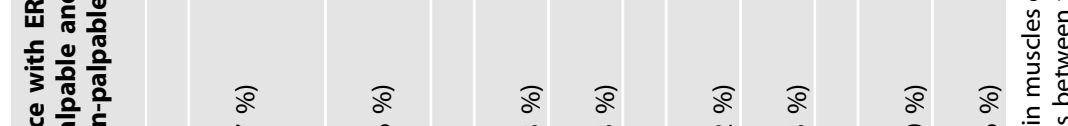

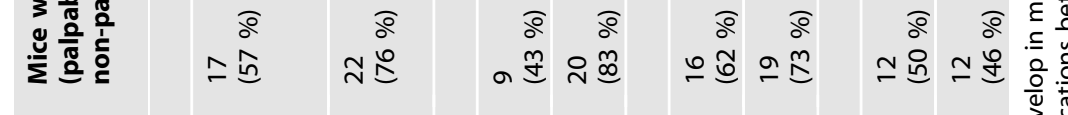

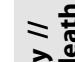

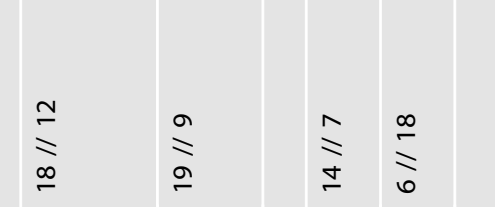

离

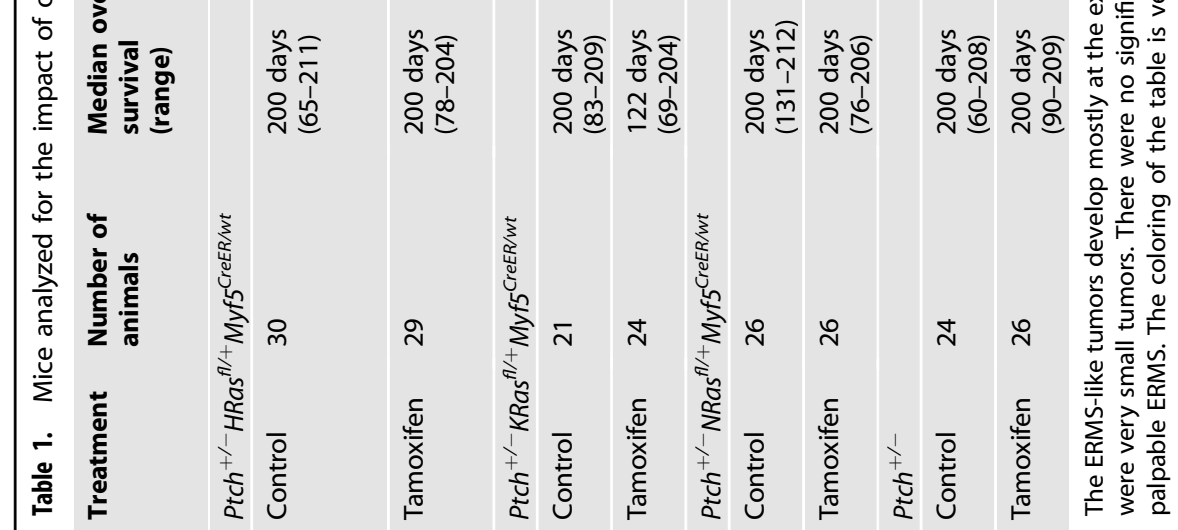

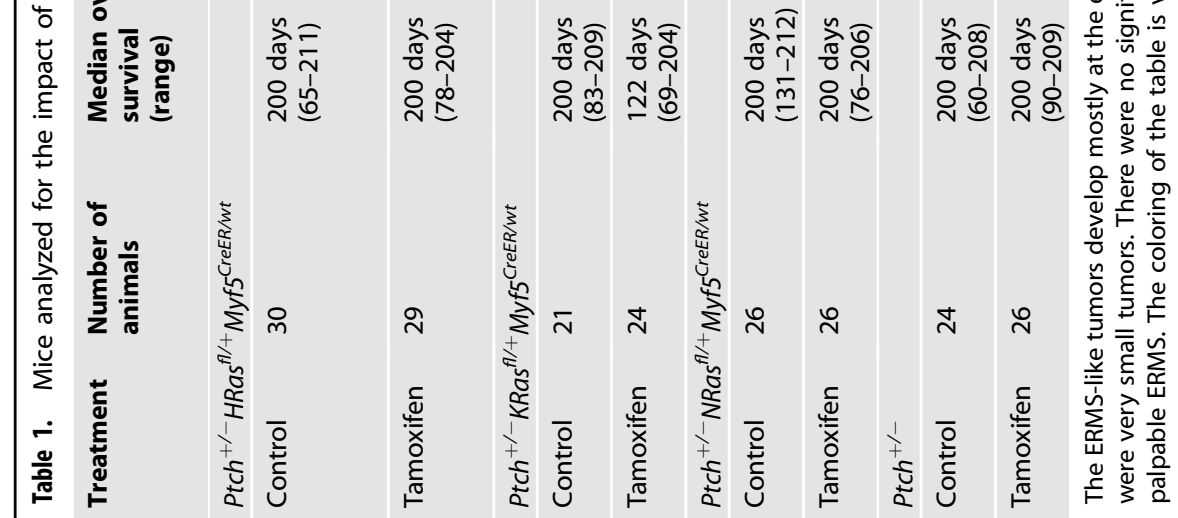

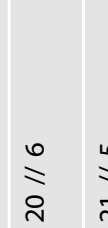

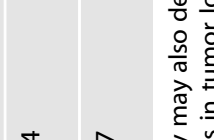

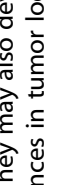

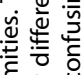

気苞这 
A Ptch $^{+/-}$HRas $^{f l /+}$ Myf5 ${ }^{\mathrm{CreER} / w t}$
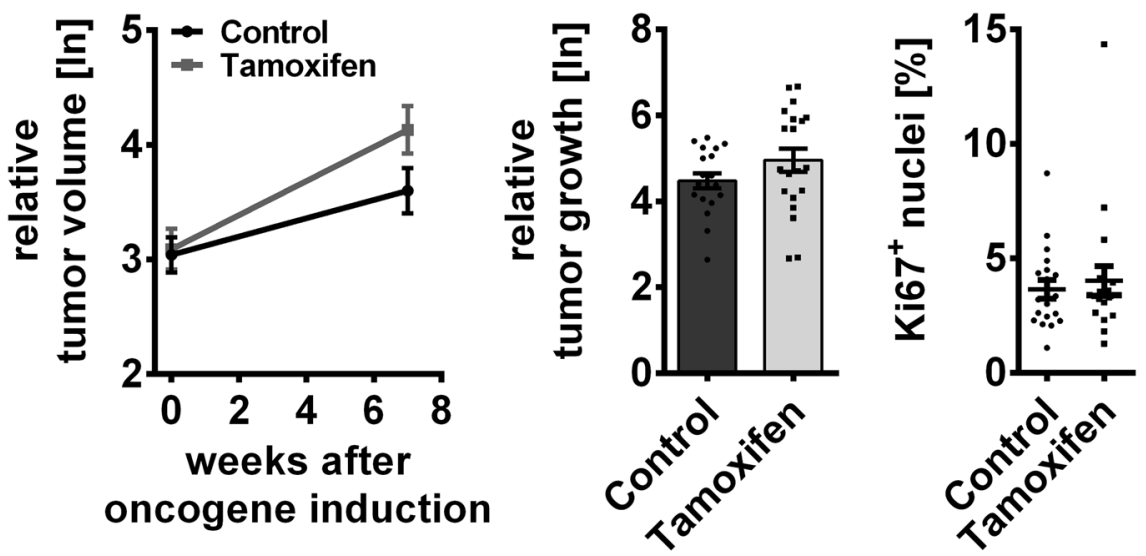

B Ptch $^{+/-}$KRas $^{f l /+} M y f 5^{C r e E R / w t}$
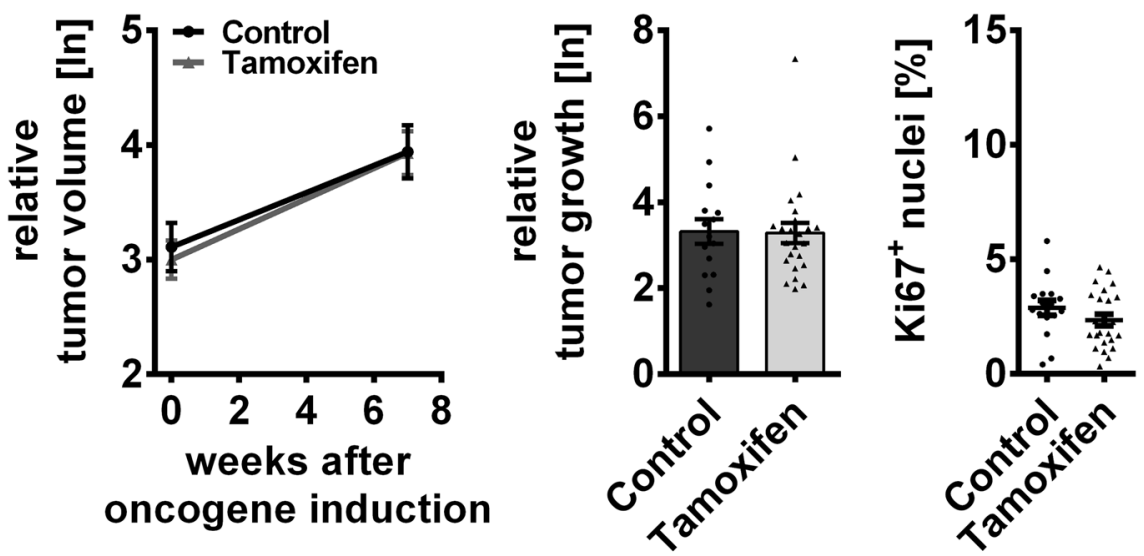

C Ptch $^{+/-}$Ras $^{f l /+}$ Myf5 ${ }^{\mathrm{CreER} / w t}$
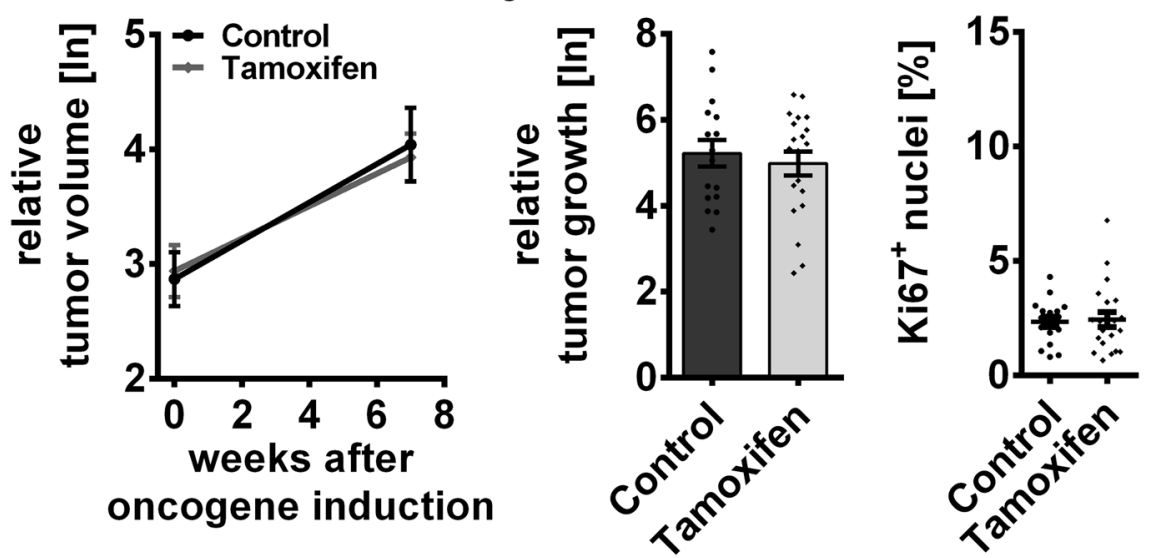

Fig. 6 Influence of oncRAS mutations on established ERMS in Ptch ${ }^{+/-}$mice. A-C ERMS growth monitored by $\mu C T$ measurements before and 7 weeks after tamoxifen-mediated induction of the oncRAS mutations in A Ptch ${ }^{+/-}$RRas $^{f /+}$ Myf5 $^{\text {CreER/wt }}$ B Ptch ${ }^{+/-}$KRas $^{f /+}$ Myf5 $^{\text {CreER/wt }}$ or C Ptch $^{+1-} \mathrm{NRas}^{f / /} \mathrm{Myf5}^{\mathrm{CreER} / \mathrm{wt}}$ mice. At least 12 animals were analyzed per cohort. Left: mean relative tumor volumes before and 7 weeks after

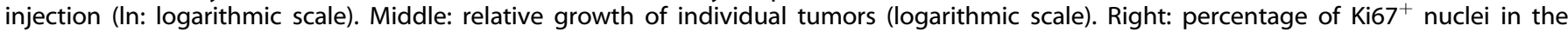
tumors. Solvent-treated mice served as controls. All ERMS of the same mouse were analyzed as individual tumors. Dots: results for individual tumors. Statistical analyses of mean tumor growth or the individual tumor growth and the percentage of Ki67+ nuclei were done by Student's $t$-tests or non-parametric $t$-tests (Mann-Whitney), respectively.

to the respective mutation, may result in a more aggressive ERMS growth. This scenario would be similar to many other cancerrelated mutations that can show cell type, cell differentiation and tumorigenesis-stage specificity (for review see ref. [45]).
Similar to LOH of $11 \mathrm{p} 15.5$, oncRAS mutations are generally considered as ERMS founding lesions [7]. However, our data on human cell lines show that oncRAS mutations also function as "advantageous mutations" for already established ERMS cells. 
A ERMS precursor
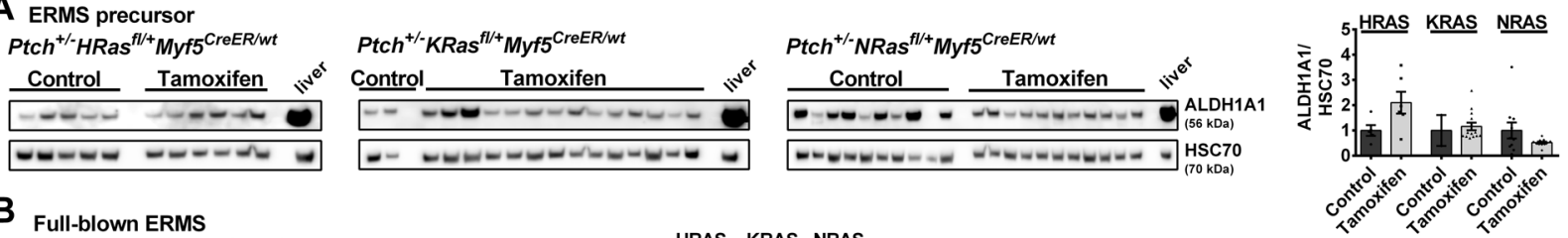

B Full-blown ERMS

Ptch $^{+/}$oncRas ${ }^{l /+}$ Myf5 ${ }^{\text {CreER/wt }}$

HRAS KRAS NRAS

Control Tam. Control Tam. Control Tam.
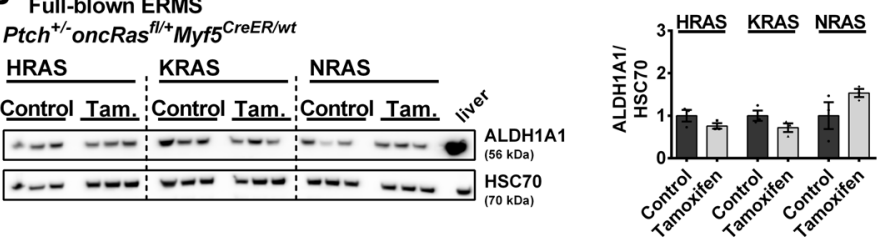

C ${ }_{\text {Ptch }}{ }^{+/}$HRas $^{f /+} M y f 5^{\text {CreER/wt }}$ ERMS precursor
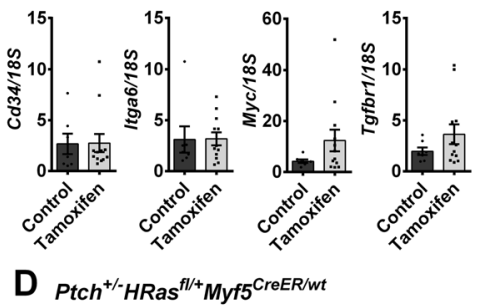

Ptch $^{+/ /}$KRas $^{f / /}$ Myf5 CreER/wt ERMS precursor
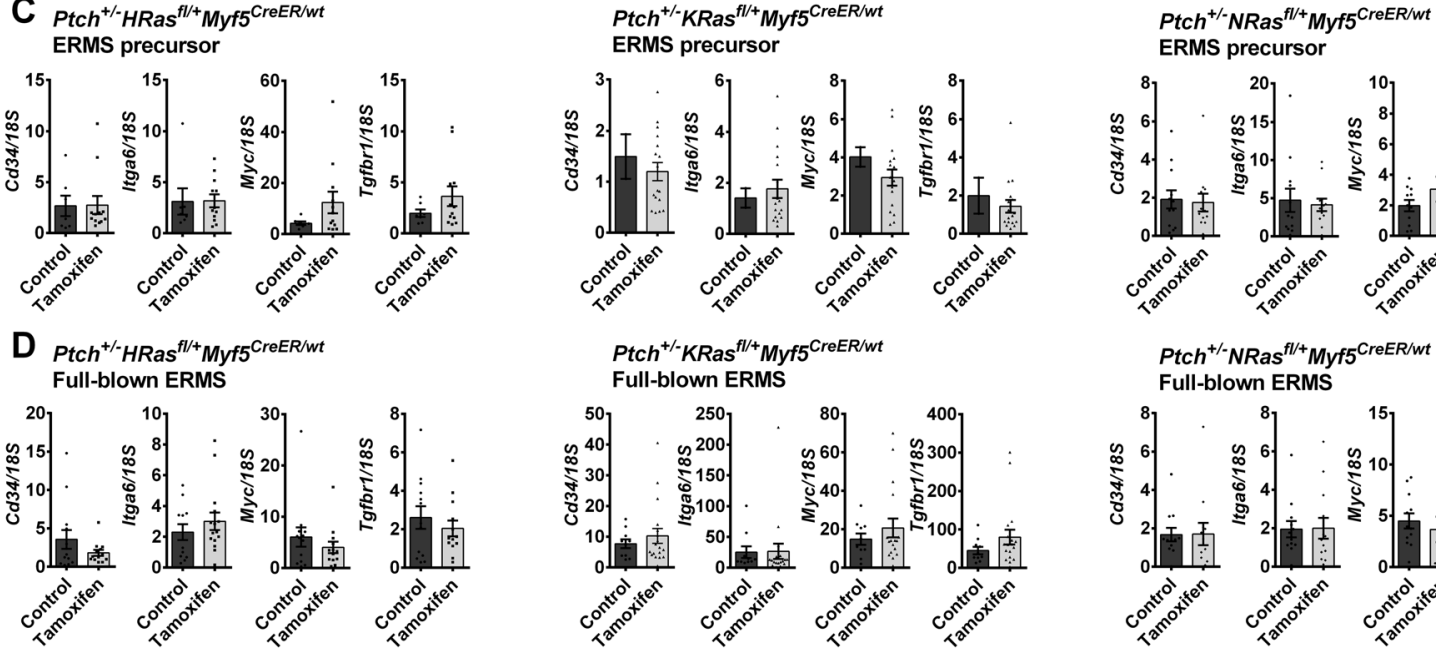

\section{Ptch $^{+/-}$KRas ${ }^{f / /}$ Myf5 $5^{\text {CreER/wt }}$}

Full-blown ERMS
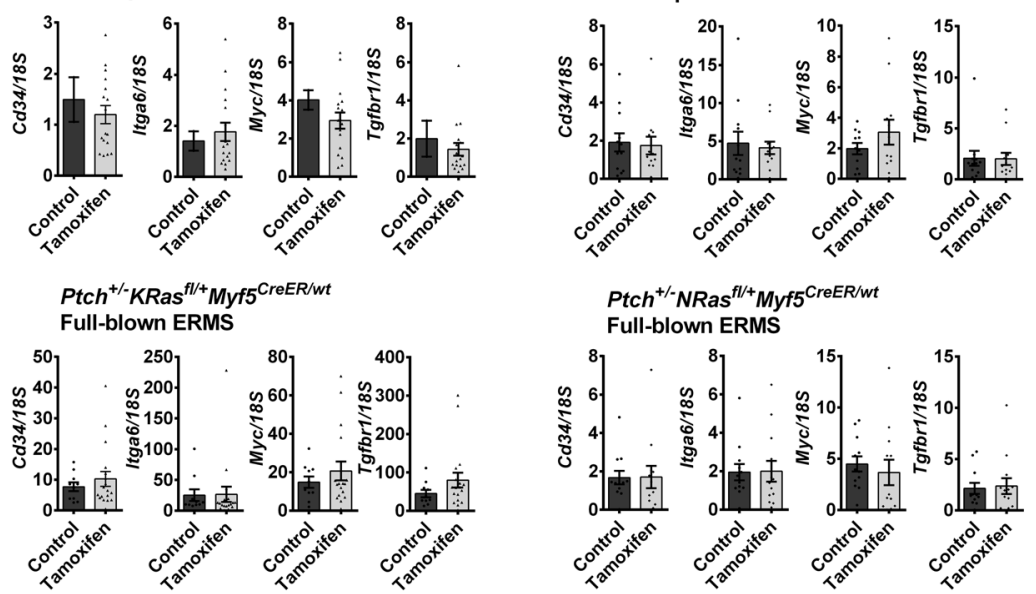

\section{Ptch $^{+/-N R a s^{f l /+} M y f 5}{ }^{\text {CreER/wt }}$}

Full-blown ERMS

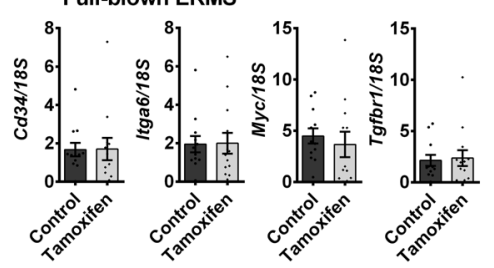

Fig. 7 Expression of stem cell markers in oncRAS-expressing ERMS from Ptch ${ }^{+/-}$mice. A, B Representative western blots (left) and respective densitometric analyses (right) for ALDH1A1 protein levels of tamoxifen-treated Ptch ${ }^{+/-}$HRas $^{\mathrm{fl} /+} \mathrm{Myf5}^{\mathrm{CreER} / \mathrm{wt}}$, Ptch ${ }^{+/-} \mathrm{KRas}^{\mathrm{fl} /+} \mathrm{Myf5}^{\mathrm{CreER} /}$ wt , or Ptch ${ }^{+/-} \mathrm{NRas}^{f / /} \mathrm{Myf5}^{\mathrm{CreER} / \text { wt }}$ mice with oncRAS mutations induced at the precursor (A) or the full-blown stage (B) in comparison to control mice. C, D qRT-PCR analyses of Cd34, Itga6, Myc, and Tgfbr1 in ERMS with oncRAS induction at the ERMS precursor stage (C) or at the full-blown ERMS stage (D) shown as fold expression of the same gene in normal muscle of the same mouse, which was set to 1 . Dots: values from individual tumors. Statistical evaluation was done by non-parametric $t$-tests (Mann-Whitney). Bars: mean \pm SEM.

In addition, the mutations seem to enlarge the $A L D H^{\text {high }}$ populations that potentially belong to cancer-initiating cells in sarcoma [34, 46]. Furthermore, oncRAS mutations induce the expression of several CSC markers in RUCH-2 cells. Therefore, it is possible that oncRAS can enhance ERMS development and proliferation by pushing the cells into a CSC phenotype. However, this is hypothetical and needs verification.

We currently do not know why oncRAS mutations do not affect growth of full-blown ERMS in $\mathrm{Ptch}^{+/-}$mice. Similar to human ERMS cell lines that are also derived from full-blown ERMS, ERMS of Ptch ${ }^{+/-}$mice contain ALDH1A1 $1^{+}$subpopulations and express CSC markers, which however are not modulated by oncRAS. Again, this discrepancy may reflect species-specific differences in tumor pathobiology or could be related to active Hh signaling. Yet it is also well possible that the full-blown murine tumors grow independently of RAS signaling. We currently also do not know if ERMS precursor lesions of Ptch $^{+/-}$mice contain cells that could be specifically targeted by oncRAS. However, this seems likely because oncHRAS and oncKRAS germline mutations per se do not lead to ERMS, at least not in the mouse [14-17]. Therefore, both mutations must have affected growth of already existing ERMS precursor lesions in $\mathrm{Ptch}^{+/-}$mice. This argues for the intriguing possibility that oncRAS mutations are not the ERMS-initiating event but are advantageous for already initiated ERMS lesions.

If oncRAS mutations are not the ERMS-initiating event, the alternative could be $\mathrm{LOH}$ of $11 \mathrm{p} 15.5$, which is much more common and occurs in almost all ERMS (e.g., 24/25 fusion-negative RMS described by ref. [7]). LOH of $11 p 15.5$ is usually accompanied by uniparental di- to pentasomy [7] with loss of maternal genetic information and duplication of the paternal one, which results in IGF2 overexpression [47, 48]. Interestingly, LOH or uniparental disomy of $11 \mathrm{p} 15.5$ are also seen in ERMS from patients with Costello Syndrome or Noonan Syndrome [13, 49, 50]. Together with the facts that (i) oncRas mutations in mice do not result in ERMS, (ii) almost all ERMS overexpress IGF2, and (iii) lgf2 is indispensable for ERMS formation, at least in mice [51], it is possible that $\mathrm{LOH}$ of $11 \mathrm{p} 15.5$, and not an oncRAS mutation, is the ERMS-initiating event. Whether this is true or not remains to be analyzed in future studies.

\section{MATERIALS AND METHODS}

\section{Cell lines}

The human ERMS cell lines RUCH-2 and TE617.T were transduced with pMSCV puro vector (Clontech, \#634401) containing RAS sequences derived from pCaggs-NRAS ${ }^{G 12 V}$ [52], a KRAS ${ }^{G 12 V}$ plasmid [40] or pBabe puro $H R A S^{G 12 V}$ (Addgene plasmid \#905).

Source of cell lines, culture conditions, and detailed experimental procedures for standard methods (e.g., BrdU incorporation assay, WST-1 and Aldefluor assays, flow cytometry, xenografting, and analysis of gene or protein expression) are described in the Supplementary Material and Methods section.

\section{Animal experiments}

Studies have been approved by the Lower Saxony State Office for Consumer Protection and Food Safety (file numbers 33.14.42502-04-13/ 
1284, 33.9-42502-04-12/0805, and 33.14.42502-04-17/2534). Numbers of used animals are included in the respective figures or tables.

We used nude (Crl:NU(NCr)-Foxn 1 ${ }^{\text {nu }}$, Charles River), Ptch ${ }^{+/}$[35] Myf5 $^{\text {CreER }}$ [37], Rosa26R-LacZ (R26R, JAX stock \#002073, [53]) mice, and HRAS (FR-HRASG12V; [16]), KRAS (LSL-K-RASG12D; [54]) or NRAS (NRAS LSLG12D [44]) - collectively named oncRas ${ }^{A /+}$-mice for the studies. Detailed breedings, cre-recombination upon tamoxifen injection, tumor monitoring, $\mu C T$ measurements, and immunohistochemical analyses are described in the Supplementary Material and Methods section. Utilized oligonucleotides and antibodies are depicted in Supplementary Tables S1 and S2, respectively.

\section{Statistical analyses}

Statistical tests done by Microsoft ${ }^{\circledR}$ Excel $^{\circledR} 2016$ or GraphPad Prism 6 are given in the respective figure legends. Data were considered significant when $p<0.05$. All tests were two-sided and $p$-values were not corrected for multiple testing.

\section{REFERENCES}

1. Siegel RL, Miller KD, Jemal A. Cancer statistics, 2020. CA: Cancer J Clin. 2020;70:7-30.

2. Dasgupta R, Fuchs J, Rodeberg D. Rhabdomyosarcoma. Semin Pediatr Surg (Rev). 2016;25:276-83

3. Soleimani VD, Rudnicki MA. New insights into the origin and the genetic basis of rhabdomyosarcomas. Cancer Cell (Comment). 2011;19:157-9.

4. Boscolo Sesillo F, Fox D, Sacco A. Muscle stem cells give rise to rhabdomyosarcomas in a severe mouse model of duchenne muscular dystrophy. Cell Rep. 2019;26:689-701. e686

5. Rubin BP, Nishijo K, Chen HI, Yi X, Schuetze DP, Pal R, et al. Evidence for an unanticipated relationship between undifferentiated pleomorphic sarcoma and embryonal rhabdomyosarcoma. Cancer Cell. 2011;19:177-91.

6. Genadry KC, Pietrobono S, Rota R, Linardic CM. Soft tissue sarcoma cancer stem cells: an overview. Front Oncol (Rev). 2018;8:475.

7. Chen L, Shern JF, Wei JS, Yohe ME, Song YK, Hurd L, et al. Clonality and evolutionary history of rhabdomyosarcoma. PLoS Genet. 2015;11:e1005075.

8. Ignatius MS, Chen E, Elpek NM, Fuller AZ, Tenente IM, Clagg R, et al. In vivo imaging of tumor-propagating cells, regional tumor heterogeneity, and dynamic cell movements in embryonal rhabdomyosarcoma. Cancer Cell. 2012;21:680-93.

9. Paulson V, Chandler G, Rakheja D, Galindo RL, Wilson K, Amatruda JF, et al. Highresolution array CGH identifies common mechanisms that drive embryonal rhabdomyosarcoma pathogenesis. Genes Chromosomes Cancer. 2011;50:397-408.

10. Chen X, Stewart E, Shelat AA, Qu C, Bahrami A, Hatley M, et al. Targeting oxidative stress in embryonal rhabdomyosarcoma. Cancer Cell. 2013;24:710-24.

11. Shern JF, Chen L, Chmielecki J, Wei JS, Patidar R, Rosenberg M, et al. Comprehensive genomic analysis of rhabdomyosarcoma reveals a landscape of alterations affecting a common genetic axis in fusion-positive and fusion-negative tumors. Cancer Disco. 2014;4:216-31.

12. Langenau DM, Keefe MD, Storer NY, Guyon JR, Kutok JL, Le X, et al. Effects of RAS on the genesis of embryonal rhabdomyosarcoma. Genes Dev. 2007;21:1382-95.

13. Kratz CP, Rapisuwon S, Reed $\mathrm{H}$, Hasle $\mathrm{H}$, Rosenberg PS. Cancer in noonan, costello, cardiofaciocutaneous and LEOPARD syndromes. Am J Med Genet Part C, Semin Med Genet. 2011;157C:83-89.

14. Tsumura H, Yoshida T, Saito H, Imanaka-Yoshida K, Suzuki N. Cooperation of oncogenic K-ras and p53 deficiency in pleomorphic rhabdomyosarcoma development in adult mice. Oncogene. 2006;25:7673-9.

15. Guerra C, Mijimolle N, Dhawahir A, Dubus P, Barradas M, Serrano M, et al. Tumor induction by an endogenous $\mathrm{K}$-ras oncogene is highly dependent on cellular context. Cancer Cell. 2003:4:111-20.

16. Chen X, Mitsutake N, LaPerle K, Akeno N, Zanzonico P, Longo VA, et al. Endogenous expression of $\operatorname{Hras}(\mathrm{G} 12 \mathrm{~V})$ induces developmental defects and neoplasms with copy number imbalances of the oncogene. Proc Natl Acad Sci USA. 2009;106:7979-84.

17. Wang J, Liu Y, Li Z, Wang Z, Tan LX, Ryu MJ, et al. Endogenous oncogenic Nras mutation initiates hematopoietic malignancies in a dose- and cell typedependent manner. Blood. 2011;118:368-79.

18. Zibat A, Missiaglia E, Rosenberger A, Pritchard-Jones K, Shipley J, Hahn H, et al. Activation of the hedgehog pathway confers a poor prognosis in embryonal and fusion gene-negative alveolar rhabdomyosarcoma. Oncogene. 2010;29:6323-30.

19. Pressey JG, Anderson JR, Crossman DK, Lynch JC, Barr FG. Hedgehog pathway activity in pediatric embryonal rhabdomyosarcoma and undifferentiated sarcoma: A report from the Children's Oncology Group. Pediatr Blood Cancer. 2011;57:930-8.
20. Almazan-Moga A, Zarzosa P, Molist C, Velasco P, Pyczek J, Simon-Keller K. et al. Ligand-dependent Hedgehog pathway activation in Rhabdomyosarcoma: the oncogenic role of the ligands. Br J Cancer. 2017;117:1314-25.

21. Pasca di Magliano $M$, Hebrok M. Hedgehog signalling in cancer formation and maintenance. Nat Rev Cancer. 2003;3:903-11.

22. Hettmer S, Teot LA, Kozakewich $\mathrm{H}$, Werger AM, Davies $\mathrm{KJ}$, Fletcher $C D$, et al. Myogenic tumors in nevoid Basal cell carcinoma syndrome. J Pediatr Hematol Oncol. 2015;37:147-9.

23. Hahn H, Wojnowski L, Zimmer AM, Hall J, Miller G, Zimmer A. Rhabdomyosarcomas and radiation hypersensitivity in a mouse model of Gorlin syndrome. Nat Med. 1998;4:619-22.

24. Aberger F, Kern D, Greil R, Hartmann TN. Canonical and noncanonical Hedgehog/ GLI signaling in hematological malignancies. Vitam Horm. 2012;88:25-54.

25. Stecca B, Ruiz IAA. Context-dependent regulation of the GLI code in cancer by HEDGEHOG and non-HEDGEHOG signals. J Mol Cell Biol. 2010;2:84-95.

26. Geyer N, Ridzewski R, Bauer J, Kuzyakova M, Dittmann K, Dullin C. et al. Different response of ptch mutant and ptch wildtype rhabdomyosarcoma toward SMO and PI3K inhibitors. Front Oncol. 2018;8:396

27. Ridzewski R, Rettberg D, Dittmann K, Cuvelier N, Fulda S, Hahn H. Hedgehog inhibitors in rhabdomyosarcoma: a comparison of four compounds and responsiveness of four cell lines. Front Oncol. 2015;5:130.

28. Pietrobono S, Gagliardi S, Stecca B. Non-canonical hedgehog signaling pathway in cancer: activation of GLI transcription factors beyond smoothened. Front Genet (Rev). 2019;10:556.

29. Chardin P, Yeramian P, Madaule P, Tavitian A. N-ras gene activation in the RD human rhabdomyosarcoma cell line. Int J Cancer. 1985;35:647-52.

30. Barretina J, Caponigro G, Stransky N, Venkatesan K, Margolin AA, Kim S, et al. The cancer cell line encyclopedia enables predictive modelling of anticancer drug sensitivity. Nature. 2012;483:603-7.

31. Dhawan P, Richmond A. A novel NF-kappa B-inducing kinase-MAPK signaling pathway up-regulates NF-kappa B activity in melanoma cells. J Biol Chem. 2002;277:7920-8.

32. Yujiri T, Sather $\mathrm{S}$, Fanger GR, Johnson GL. Role of MEKK1 in cell survival and activation of JNK and ERK pathways defined by targeted gene disruption. Science. 1998;282:1911-4.

33. Moon BS, Jeong WJ, Park J, Kim TI, Min do S, Choi KY. Role of oncogenic K-Ras in cancer stem cell activation by aberrant Wnt/beta-catenin signaling. J Natl Cancer Inst. 2014;106:djt373.

34. Nakahata K, Uehara S, Nishikawa S, Kawatsu M, Zenitani M, Oue T, et al. Aldehyde dehydrogenase 1 (ALDH1) is a potential marker for cancer stem cells in embryonal rhabdomyosarcoma. PLoS ONE. 2015;10:e0125454.

35. Zibat A, Uhmann A, Nitzki F, Wijgerde M, Frommhold A, Heller T, et al. Time-point and dosage of gene inactivation determine the tumor spectrum in conditional Ptch knockouts. Carcinogenesis. 2009;30:918-26.

36. Nitzki F, Zibat A, Frommhold A, Schneider A, Schulz-Schaeffer W, Braun T, et al. Uncommitted precursor cells might contribute to increased incidence of embryonal rhabdomyosarcoma in heterozygous Patched1-mutant mice. Oncogene. 2011;30:4428-36.

37. Biressi S, Bjornson CR, Carlig PM, Nishijo K, Keller C, Rando TA. Myf5 expression during fetal myogenesis defines the developmental progenitors of adult satellite cells. Developmental Biol. 2013;379:195-207.

38. Tostar U, Toftgard R, Zaphiropoulos PG, Shimokawa T. Reduction of human embryonal rhabdomyosarcoma tumor growth by inhibition of the hedgehog signaling pathway. Genes Cancer. 2010;1:941-51.

39. Zhao X, Ponomaryov T, Ornell KJ, Zhou P, Dabral SK, Pak E, et al. RAS/MAPK activation drives resistance to smo inhibition, metastasis, and Tumor evolution in shh pathway-dependent tumors. Cancer Res. 2015;75:3623-35.

40. Lauth M, Bergstrom A, Shimokawa T, Tostar U, Jin Q, Fendrich V, et al. DYRK1Bdependent autocrine-to-paracrine shift of Hedgehog signaling by mutant RAS. Nat Struct Mol Biol. 2010;17:718-25.

41. Mercer SE, Ewton DZ, Shah S, Naqvi A, Friedman E. Mirk/Dyrk1b mediates cell survival in rhabdomyosarcomas. Cancer Res. 2006;66:5143-50.

42. Ashford AL, Dunkley TP, Cockerill M, Rowlinson RA, Baak LM, Gallo R, et al. Identification of DYRK1B as a substrate of ERK1/2 and characterisation of the kinase activity of DYRK1B mutants from cancer and metabolic syndrome. Cell Mol life Sci. 2016;73:883-900.

43. Yohe ME, Gryder BE, Shern JF, Song YK, Chou HC, Sindiri S. et al. MEK inhibition induces MYOG and remodels super-enhancers in RAS-driven rhabdomyosarcoma. Sci Transl Med. 2018;10:eaan4470

44. Haigis KM, Kendall KR, Wang Y, Cheung A, Haigis MC, Glickman JN, et al. Differential effects of oncogenic K-Ras and N-Ras on proliferation, differentiation and tumor progression in the colon. Nat Genet. 2008;40:600-8.

45. Sieber OM, Tomlinson SR, Tomlinson IP. Tissue, cell and stage specificity of (epi) mutations in cancers. Nat Rev Cancer (Rev). 2005;5:649-55. 
4966

46. Martinez-Cruzado L, Tornin J, Santos L, Rodriguez A, Garcia-Castro J, Moris F, et al. Aldh1 expression and activity increase during tumor evolution in sarcoma cancer stem cell populations. Sci Rep. 2016;6:27878.

47. Scrable H, Cavenee W, Ghavimi F, Lovell M, Morgan K, Sapienza C. A model for embryonal rhabdomyosarcoma tumorigenesis that involves genome imprinting. Proc Natl Acad Sci USA. 1989;86:7480-4.

48. Zhan S, Shapiro DN, Helman $\sqcup$. Activation of an imprinted allele of the insulin-like growth factor II gene implicated in rhabdomyosarcoma. J Clin Investig. 1994;94:445-8.

49. Robbins KM, Stabley DL, Holbrook J, Sahraoui R, Sadreameli A, Conard K, et al. Paternal uniparental disomy with segmental loss of heterozygosity of chromosome 11 are hallmark characteristics of syndromic and sporadic embryonal rhabdomyosarcoma. Am J Med Genet Part A. 2016;170:3197-206.

50. Jongmans MC, Hoogerbrugge PM, Hilkens L, Flucke U, van der Burgt I, Noordam $\mathrm{K}$, et al. Noonan syndrome, the SOS1 gene and embryonal rhabdomyosarcoma. Genes Chromosomes Cancer. 2010;49:635-41.

51. Hahn H, Wojnowski L, Specht K, Kappler R, Calzada-Wack J, Potter D, et al. Patched target lgf2 is indispensable for the formation of medulloblastoma and rhabdomyosarcoma. J Biol Chem. 2000;275:28341-4.

52. Kang TW, Yevsa T, Woller N, Hoenicke L, Wuestefeld T, Dauch D, et al. Senescence surveillance of pre-malignant hepatocytes limits liver cancer development. Nature. 2011;479:547-51.

53. Soriano P. Generalized lacZ expression with the ROSA26 Cre reporter strain. Nat Genet. 1999;21:70-71.

54. Tuveson DA, Shaw AT, Willis NA, Silver DP, Jackson EL, Chang S, et al. Endogenous oncogenic K-ras(G12D) stimulates proliferation and widespread neoplastic and developmental defects. Cancer Cell. 2004;5:375-87.

\section{ACKNOWLEDGEMENTS}

We thank Anke Frommhold, Ina Heß (Institute of Human Genetics, University Medical Center Goettingen) and Lena Ries (Department of Haematology/Medical Oncology, University Medical Center Goettingen) for excellent technical assistance. We are grateful to the animal caretakers and Dr. Verena Reupke for excellent animal care (ZTE, University Medical Center Goettingen), Sabrina Becker (Cell Sorting Unit, University Medical Center Goettingen), Michael Engelke and Jens Löber (Institute for Cellular and Molecular Immunology, University Medical Center Goettingen) for providing support for flow cytometry experiments, Christina Malowsky and Sarah Garbode (Institute for Diagnostic and Interventional Radiology, University Medical Center Goettingen) for assistance at the $\mu \mathrm{CT}$ and Matthias Lauth (Center for Tumor Biology and Immunology, School of Medicine, University Marburg) and Tae-Won Kang (Helmholtz Centre for Infection Research, Braunschweig) for sharing plasmids.

\section{AUTHOR CONTRIBUTIONS}

Conceptualization: JB, NC, NR, FN, AU, and HH; formal analysis: JB, NC, NR, KSK, AR, HUS, WSS, $A U$, and $\mathrm{HH}$; funding acquisition: $\mathrm{KSK}, \mathrm{FA}$, and $\mathrm{HH}$; investigation: JB, NC, NR,
KSK, NG, DSB, and A.U.; methodology: JB, NC, NR, KSK, FN, DPE, AR, CD, FA, and HH; project administration: $H H$; resources: $T A R, S B, J A F, D S$, and $H H$; software: $C D$ and $A U$; supervision: $\mathrm{FN}, \mathrm{FA}, \mathrm{AU}$, and $\mathrm{HH}$; visualization: $J \mathrm{~B}, \mathrm{AU}$; writing -original draft: $J \mathrm{~B}, \mathrm{HH}$; writing-review \& editing: JB, $\mathrm{AU}$, and $\mathrm{HH}$.

\section{FUNDING}

This work was supported by grants HA 2197/9-1 and HA 2197/9-2 from the German Research Foundation to $\mathrm{HH}$, by grant 2017.110.1 of the Wilhelm Sander foundation to HH. and KSK. and by grants P25629 and W1213 of the Austrian Science Fund (FWF) to FA. Open Access funding enabled and organized by Projekt DEAL.

\section{COMPETING INTERESTS}

The authors declare no competing interests.

\section{ADDITIONAL INFORMATION}

Supplementary information The online version contains supplementary material available at https://doi.org/10.1038/s41388-021-01904-4.

Correspondence and requests for materials should be addressed to H.H.

Reprints and permission information is available at http://www.nature.com/ reprints

Publisher's note Springer Nature remains neutral with regard to jurisdictional claims in published maps and institutional affiliations.

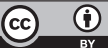

Open Access This article is licensed under a Creative Commons Attribution 4.0 International License, which permits use, sharing, adaptation, distribution and reproduction in any medium or format, as long as you give appropriate credit to the original author(s) and the source, provide a link to the Creative Commons license, and indicate if changes were made. The images or other third party material in this article are included in the article's Creative Commons license, unless indicated otherwise in a credit line to the material. If material is not included in the article's Creative Commons license and your intended use is not permitted by statutory regulation or exceeds the permitted use, you will need to obtain permission directly from the copyright holder. To view a copy of this license, visit http://creativecommons. org/licenses/by/4.0/.

(c) The Author(s) 2021 